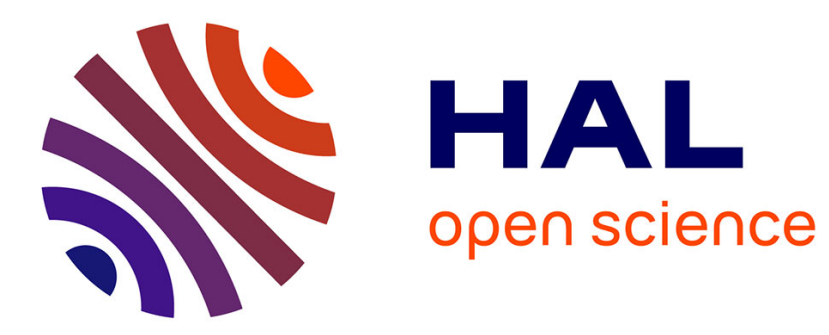

\title{
ViCoV: Efficient Video Streaming for Cognitive Radio VANET
}

\author{
Abbas Bradai, Toufik Ahmed, Abderrahim Benslimane
}

\section{To cite this version:}

Abbas Bradai, Toufik Ahmed, Abderrahim Benslimane. ViCoV: Efficient Video Streaming for Cognitive Radio VANET. Vehicular Communications, 2014, pp.1-18. 10.1016/j.vehcom.2014.05.002 . hal00997616v2

\section{HAL Id: hal-00997616 https://hal.science/hal-00997616v2}

Submitted on 28 Sep 2014

HAL is a multi-disciplinary open access archive for the deposit and dissemination of scientific research documents, whether they are published or not. The documents may come from teaching and research institutions in France or abroad, or from public or private research centers.
L'archive ouverte pluridisciplinaire HAL, est destinée au dépôt et à la diffusion de documents scientifiques de niveau recherche, publiés ou non, émanant des établissements d'enseignement et de recherche français ou étrangers, des laboratoires publics ou privés. 


\title{
ViCoV: Efficient Video Streaming for Cognitive Radio VANET
}

\author{
Abbas Bradai and Toufik Ahmed \\ CNRS-LaBRI University of Bordeaux \\ 351 , Cours de la libération \\ Talence, 33405 \\ \{bradai, tad\}@labri.fr
}

\author{
Abderrahim Benslimane \\ LIA/CERI University of Avignon \\ 339, Chemin des Meinajaries \\ Avignon, 84911 cedex 9 \\ benslimane@ieee.org
}

\begin{abstract}
One of the major challenges facing vehicular ad-hoc communication is to ensure efficient services delivery under different network conditions. In this context, video communication is envisioned by the scientific and industrial communities to be of high benefit for traffic management as well as for providing value-added entertainment and advertising services. In this paper, we propose a new protocol for efficient VIdeo streaming over COgnitive radio VANETs (ViCoV), a video streaming solution which broadcasts safety and entrainment content in both fully and intermittently connected networks under different traffic conditions. First, ViCoV selects the best available dedicated or Cognitive Radio (CR) channels to disseminate the content. Then, it carefully choses a minimum sub-set of rebroadcaster nodes to reduce interferences and to achieve high video quality. The CR channels are selected based on their stability over the time, whereas the rebroadcaster nodes are selected based on a new centrality metric, inspired from the Social Network Analysis (SNA), called dissemination capacity. Through simulations, we compared $\mathrm{ViCoV}$ with the multichannel vehicular communication standard IEEE 1609.4 and two pioneering video streaming mechanisms over VANET. The performance evaluation shows that ViCoV outperforms the abovementioned mechanisms in terms of providing higher video delivery ratio, lower end-to-end transmission delay and lower frame loss ratio in both fully and intermittently connected networks.
\end{abstract}

Keywords: VANET; QoS; Multimedia; Content delivery; Time series; Social network Analysis (SNA)

\section{INTRODUCTION}

Vehicular ad-hoc networks (VANETs) belong to the general class of mobile ad-hoc networks with vehicles acting as fast moving mobile nodes. More specifically, a VANET consists of on-board-units (OBUs) installed on the vehicles and road-side units (RSUs) deployed along sides of the urban roads/highways which facilitate both vehicle-to-vehicle (V2V) communications and vehicle-toinfrastructure (V2I) communications. Intelligent transportation systems (ITS) for vehicular ad-hoc networks (VANETs) have stimulated the development of several interesting applications, such as vehicle collision warning, security distance warning, driver assistance, cooperative driving, etc. The vehicle engine provides sufficient power for intensive data processing and communications. The onboard buffer storage, positioning system, and intelligent antenna further facilitate efficient video forwarding and collaborative downloading among vehicles or from/to RSUs. 


\section{Motivations}

Besides the traditional applications of VANET, such as accident alert and traffic information exchanged as plain text, the scientific and industrial communities envisage video communication within vehicular networks to be of major benefit for traffic management as well as to provide a value-added entertainment / advertising services. Indeed, in a road emergency, streaming a live video of the accident area allows official vehicles (police, ambulance, etc.) approaching the scene, to better understand the nature of the accident and take the right decision consequently. In addition, the distribution of multimedia content for OBUs in a certain area of interest is a promising service. Example of such services includes a local hotel broadcasting video advertising to vehicles entering the city, a travel company promoting their activities in touristic areas to passing vehicles, and highway management companies broadcasting films (for rear seat passengers) in long distance part of the highway.

A candidate exploitation scenario for video streaming in vehicular network could be broadcasting video content using RSUs, where a vehicle downloads video via license-free wireless spectrum when it is within the RSU transmission range. However, supporting video streaming services by RSUs using the license-free wireless communication is still an open issue due to the following two concerns. Firstly, the wireless channel suffers from interference, shadowing and time-varying fading, which leads to degradation of link throughput and consequently the video quality. Secondly, the RSUs deployment is highly cost which make the deployment of sufficient RSUs to cover entire roads and highways infeasible. Thus, ad-hoc V2V communication, or Vehicular Ad-hoc Networks (VANETs), are essential to ensure wide dissemination of the video in the network.

Recently, the $802.11 \mathrm{p}$ standard (WAVE) [1] was proposed, with the main concern to ensure safety communication for vehicular traffic, and then serve applications for improved mobility and reduced environmental impact. The WAVE standard allocates a dedicated time slot for emergency and safety communication, and six communication channels for other services. Furthermore, the IEEE 1609.4 multichannel standard [32] proposes a time-division scheme for DSRC radios to alternately switch between the DSRC control channel $(\mathrm{CCH})$ and the others DSRC service channels $(\mathrm{SCH})$ in order to support different applications concurrently. Studies [3][30][31] [32] show that, for both WAVE and IEEE 1609.4, the secondary services suffers from large packet delays and lack of available bandwidth in high density environment and high bandwidth demanding applications. This is due mainly to the spectrum scarceness of DSRC channels in such conditions. On the other hand, we note that there are many licensed bands, such as $400-700 \mathrm{MHz}$ range, that are used occasionally, mostly under-utilized for transmission [4].

To deal with the problem of spectrum scarceness, the FCC [21] has recently permitted the use of licensed bands by unlicensed devices. Thus, dynamic spectrum access mechanisms are investigated to address the current spectrum ineffectiveness problem. This new research field advocates the development of cognitive radio networks (CRN) in order to promote spectrum efficiency. The idea behind CRN is that non-licensed devices (called Secondary Users or SU) take advantage of the licensed bands when the licensed users (called Primary Users or PU) are not using it. Thus, the cognitive radio technology is a promising solution for video streaming over VANET networks, mainly in today's spectrum occupancy conditions. However, the task is challenging since the solution should deal with the interferences with the PU activity, and should also tackle the problem of useless redundant retransmissions of the traditional content broadcast mechanism. The ultimate goal is to ensure high quality, wide proliferation of the video stream in the network as well as low transmission delay.

Figure 1 illustrates a scenario of video streaming over VANET in dense environment using different channels. 


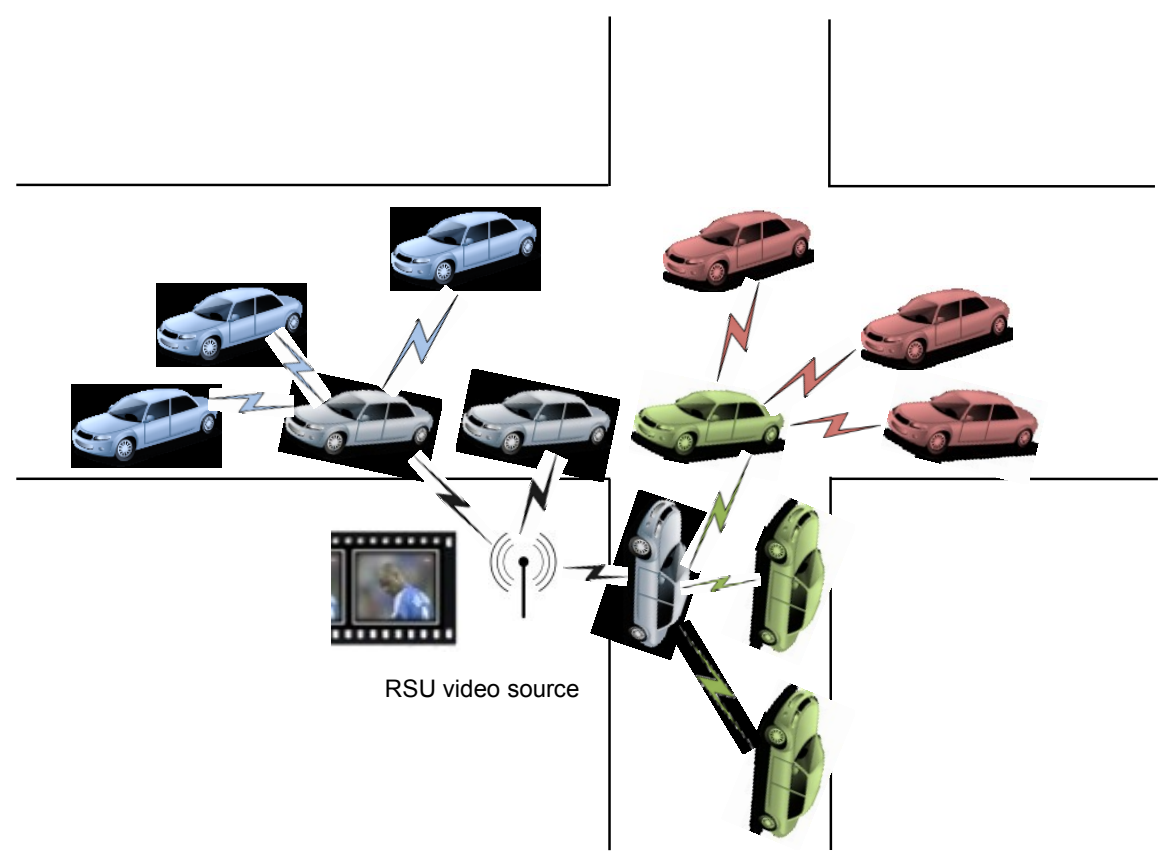

Figure 1: Example of video streaming in VANET

\section{Contributions}

In this paper, we propose $\mathrm{ViCoV}$, a distributed channel / relaying node selection mechanism for efficient VIdeo streaming over COgnitive radio VANETs (ViCoV). ViCoV is designed for both dense and sparse traffic scenarios. In dense-traffic scenario where channels are overloaded and the network suffers from high interference degree, $\mathrm{ViCoV}$ selects the best channel to disseminate the video, and a minimum sub-set of broadcasting nodes, in order to reduce interferences and perform high video quality delivery. Alternatively, in sparse traffic scenario, $\mathrm{ViCoV}$ adopt the store-carry-forward mechanism in order to increase the content delivery capability by serving vehicles missing some video content. Missing video content can be due to the intermittent connected nature of the vehicular communication or due to collisions.

Our main contributions in this paper are as follows:

1) A Channel selection mechanism for video transmission over VANET is proposed. The proposed mechanism prioritizes the safety applications messages and selects the best DSRC channel for transmission. In addition, in order to ensure efficient video transmission, the DSRC channels range is extended by selecting a Cognitive Radio (CR) channel (non DSRC channel) for transmission;

2) An Intelligent $C R$ channel selection mechanism is proposed. ViCoV selects the $C R$ channel with low PU activity, estimated using time series model. For this purpose, the PU activity is modeled as an ARMA (Auto-Regressive Moving-average) process of parameters $\alpha$ and $\beta$ corresponding to the auto regressive and the moving average component of the model;

3) A Rebroadcaster' nodes selection mechanism is proposed. The proposed mechanism selects a subset of strategic nodes to rebroadcast the content, rather than the traditional all-nodes broadcast mechanism. The selection mechanism is based on a new centrality metric called dissemination capacity DC(v), inspired from node centrality metrics of Social Network Analysis (SNA). 


\section{Paper Organization}

The paper is organized as follows. In section II, we give an overview on related work on content dissemination in VANET and cognitive radio utilization in these networks. Section III reviews some backgrounds related to cognitive radio technology as well as the time series model adopted in our solution. Section IV describes our proposed solution ViCoV in details, and section 4 discusses its performance evaluation. Finally, section VI concludes this paper.

\section{RELATED WORKS}

Recently, video dissemination in VANET has particularly attracted researcher attention and many research works have been achieved in this field. However, despite the sporadic connected nature of VANET [7], most of the proposed solutions only considers connected VANET. In addition, solutions that consider both connected and intermittently connected networks rely on either an infrastructure of RSUs to disseminate content or coordinate communication between vehicles, or on geographical information system such as GPS.

Among solutions that consider fully connected networks, we can note StreetCast [8]. StreetCast makes use of beacon suppression mechanism to reduce massive beacon messages exchanged at congested intersections. In addition, in StreetCast, RSUs are deployed in roads intersection in order to select the best vehicle to rebroadcast the message. Authors in [9] proposed Urban Multi-hop Broadcast protocol (UMB), an 802.11-based protocol, designed to suppress broadcast redundancy by selecting the furthest vehicle from the sender to acknowledge the reception of the message and rebroadcast it. UMB relies also on a set of RSUs in intersections to propagate the messages to all road directions in a fully connected scenario. The broadcast messages suppression idea was borrowed also in [10], where authors propose three techniques to suppress redundant messages: slotted-1-persistence, weighted-p-persistence and slotted-p-persistence. All these techniques do not rely on any infrastructure or on neighbors' information exchange. For instance, weighted-p-persistence technique requires that each node reforwards the message with a certain probability $\mathrm{p}$, computed as the ratio of the source node distance to the transmission range of potential rebroadcaster node. The three proposed techniques provide a good performance in terms of reachability and broadcast redundancy reduction, but only under fully connected network conditions. Along the same lines, authors in [11] propose Adaptive Information Dissemination (AID), a statistical based broadcast protocol for VANET. This protocol do not use any kind of infrastructure support neither any neighbor information. A vehicle decides to broadcast or not based only on statistics about the inter-arrival time between the received packets. A redundantly received message is not rebroadcasted, assuming that it was rebroadcasted by many other neighbors. This protocol is designed only for fully connected networks also.

In order to exploit the different DSRC channels, the standard IEEE 1609.4 [32] have been proposed. It defines a time-division pattern for DSRC channels and alternatively switches between these channels in order to support different applications simultaneously. The Standard suggests to allocate alternatively a time slot of $50 \mathrm{~ms}$ for control channel $(\mathrm{CCH})$ which conveys safety application messages and another equal time slot to service channel $(\mathrm{SCH})$ which conveys other services messages. The intuitive problem with IEEE 1609.4 is the underutilization of the spectrum. Indeed, in some communication scenarios only safety messages are exchanged in the network. However the standard requires an equal time division between the safety message channel and service channel. Consequently $50 \%$ of the transmission capacity is wasted. The same problem occurs also in the case of only-service content 
scenario. In addition, additional problems of IEEE 1609.4 are raised [32], such as the high probability of synchronized collisions at start of a channel interval $(\mathrm{CCH}$ or $\mathrm{SCH})$, the incompatibility of the standard with the multi-radio technology and its inefficiency in the case of intermittently connected networks.

Recently, some VANET protocols design has taken into consideration both connected and intermittent connected networks scenarios [12]-[16]. DV-CAST [12] and SRD [13] rely on one hop neighbors' vehicles information without any infrastructure support to disseminate video content on VANET. However, both protocols are designed to handle one directional content' dissemination. Consequently, they are exclusively exploitable in highways. This kind of protocols does not perform well in urban environment [14]. Authors in [15] propose the Data Pouring protocol for content broadcasting in urban environments. Even though it operate in both connected and intermittently connected networks, the protocol relies on RSUs at intersections to cache and rebroadcast packets to the intersecting roads. Authors in [16] propose Urban Vehicular Broadcast (UV-CAST) protocol for content dissemination in both connected and intermittently connected networks. Based on the one-hop neighbors' information, UV-CAST acts either in broadcast suppression mode or in store-carry-forward mode. In the first mode, UV-CAST uses street mapping information to check if the vehicle is at an intersection or not in order to calculate its waiting time to rebroadcast. However in store-carry-forward mode, it checks if the vehicle is a boundary vehicle. In this case, the vehicle stores and carries the message until it encounters uninformed neighbors (vehicles missing the video content). In order to identify these uniformed neighbors, UV-CAST inspects neighbors buffer maps, exchanged periodically piggybacked in beacons. We note that in this mode, a boundary vehicle immediately rebroadcasts the missing message to the neighbor vehicle without any coordination with other boundary vehicles, which results in redundant broadcasts.

Note that all the above presented protocols rely on DSRC channels, using the IEEE $802.11 \mathrm{p}$ standard for the V2V communications. However, these channels suffer from limited capacity and less support to the wide range of services projected in VANETs. This stimulated researchers [17]-[20] to envision cognitive radio technology for VANET communications. In [17], authors propose to use cognitive radio (CR) in order to increase the spectrum opportunities for $\mathrm{V} 2 \mathrm{~V}$ communication. In this solution, vehicles share information about the spectrum availability of TV channels and collaboratively decide the channel to use in each road part. The authors in [18] exploit the predictable vehicle trajectory to detect spectrum holes of the TV spectrum and dynamically decide the channel to use, in order to improve spatial reusability of spectrum. In [19], authors also propose a framework of coordinated spectrum sensing in cognitive radio for VANET, which relies on some strategic nodes to guide the sensing. Finally, authors in [20] applied Belief Propagation (BP) technique to handle the distributed spectrum sensing and to exploit redundancies in both space and time. However, we note that all these CR based approaches focus on the spectrum sensing problem in vehicular networks, without special attention to broadcast scenarios, which need coordination between vehicles to avoid collisions. In addition, all these approaches exclusively consider the connected network scenario regardless the intermittently network connected nature of VANET. Hence, to the best of our knowledge, there is no work that attempts to provide QoS guarantees for video dissemination in VANET using cognitive radio technology in fully connected and intermittently connected networks. 


\section{BACKGROUND}

In this section, we describe some background knowledge related to cognitive radio in the context of VANET and the times series model adopted in our solution.

\section{Cognitive radio in ad hoc networks}

Although ad-hoc networks can operate on different wireless standards, the currents state of the art has typically limited their action fields in the $2.4 \mathrm{GHz}, 900 \mathrm{MHz}$ and the industrial, scientific and medical (ISM) bands. With the increasing proliferation of wireless devices, these bands are more and more getting congested [20][18]. On the other hand, many licensed bands are used only over limited periods of time and their average utilization varies between $15 \%$ and $85 \%$.

The wireless spectrum licensing is currently carried for a long term periods and over vast geographical regions. So as to deal with the problem of spectrum scarceness, the FCC has recently authorized the use of licensed bands by unlicensed users, which has given rise to Cognitive Radio Networks (CRN). The main idea of CRNs is that non-licensed devices (called Secondary Users or SU) use the licensed bands when the licensed users (called Primary Users or PU) are not using it. Cognitive radio (CR) technology allows ad-hoc networks to exploit spectrum in a dynamic fashion, and it can be formally defined as [21]: A "Cognitive Radio" is a radio that can change its transmitter parameters based on interaction with the environment in which it operates.

As most of the spectrum is licensed, the challenge is to exploit the licensed spectrum without interfering with the communication of other licensed devices, as shown in Figure 2. The cognitive radio allows the use of temporarily unexploited spectrum, referred to as spectrum hole [22]. Once this band is further used by a licensed device, the SU must move to another band to avoid interferences.

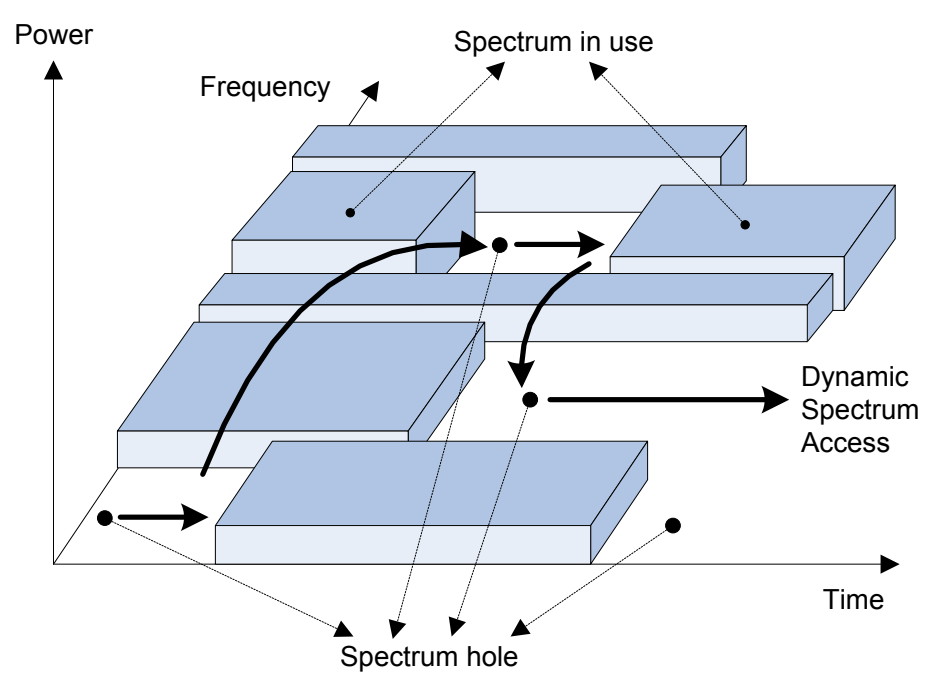

Figure 2: Dynamic spectrum access concept (source [6])

The real deployment of Cognitive radio nowadays is experiencing some regulatory and technical problems. Indeed, the radio spectrum is globally administered by the International Telecommunication Union (ITU) whereas the use of radio spectrum in each country is nationally regulated by the corresponding government agencies that have the freedom to make the spectrum available for particular use in their operational area [1]. In USA, for instance, the FCC has recently permitted the use of licensed bands by unlicensed devices, however in most countries, it is not yet permitted. Technically speaking, apart from the typical functionality of transmitting/receiving data to ensure quality of service 
in the wireless environment, the additional challenges of cognitive radios can be divided into three major categories [23]:

(1) Primary user activity sensing.

(2) Preswitching synchronization with communicating node upon successful Primary detection.

(3) Fast switching for successful rendezvous.

Many works [24][27] has addressed the implementation challenges of RC and proposed solutions. In [24], authors demonstrate that software abstraction of MAC layer implemented on commodity hardware is a feasible option for dynamic spectrum access. They implement quick and reliable spectrum sensing algorithms that exploit the inherent characteristics of the interface, as well as they implement a fast channel switching using the software abstraction. The obtained results show that high effective throughput is achievable using the implementation that they proposed. In [25], authors proposed an experimental study of distributed opportunistic spectrum access implementation. They demonstrate that, while existing hardware technologies do not provide the cognitive transceiver requirements needed to exploit Opportunistic Spectrum Access (OSA) to its full potential, suboptimal OSA approaches developed to target low-complexity transceivers can achieve significant performance improvement compared to theoretically-optimal approaches. In [26], authors demonstrated a basic CR MAC and an optimized spectrum sensing algorithm which enables secondary network formation and secondary communication in the presence of frequency hopping primary. These have been implemented and successfully tested on a SDR testbed. Thus, many effort are spends to deal with the real implementation of this technique toward a dynamic efficient spectrum access.

Along with the network architecture, CRNs can be classified into infrastructure-based CRNs and CR Ad-hoc Networks (CRAHNs) [28]. The infrastructure-based CRN relies on a central entity such as a Wi-Fi access point or cellular networks base station, while CRAHN does not have any infrastructure. Consequently, a CR device can communicate with other CR devices in ad-hoc manner on both licensed and unlicensed spectrum bands.

In the infrastructure-based CRNs, it is up to the CR central station to decide on how to avoid interference with primary users, based on the observations and analysis performed by each CR user. According to this decision, each CR user reconfigures its parameters, as illustrated in Figure 3(a). In contrast, in CRAHNs, each CR user, based on its local observation and in cooperation with other CR users, decides its communication parameters, as illustrated in Figure 3(b). Therefore, due to absence of central entity and the difficult cooperation between CR users, the selection of a common band by CR transmitters and receivers is a challenging task in multi-hop CRNs for large group content dissemination. In our solution we adopt a stochastic approach to forecast the spectrum availability in CRAHN. Each vehicle in the network forecasts the channel availability based on the time series model that we present in the following sub-section. 


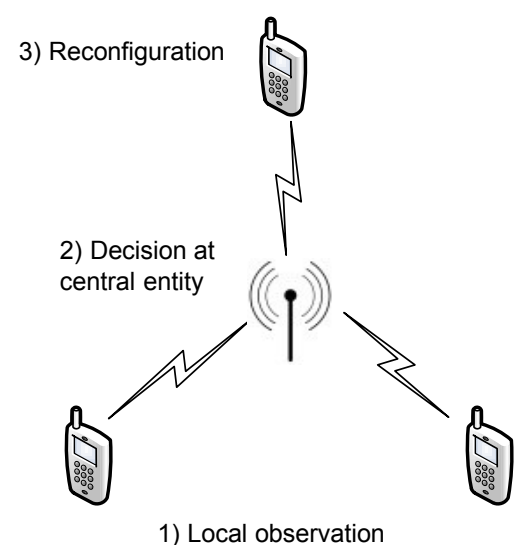

(a)

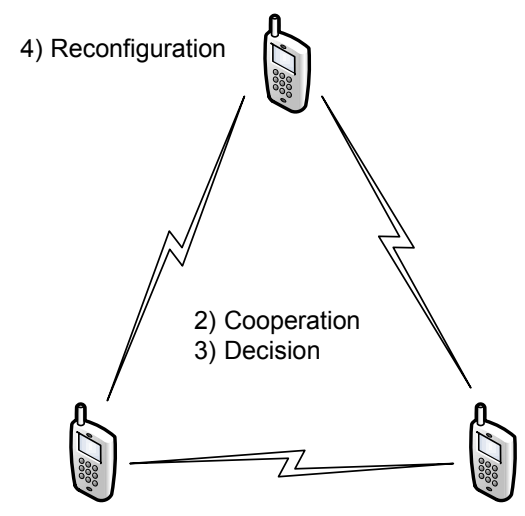

1) Local observation

(b)

Figure 3: Infrastructure-based CR network Vs CR ad-hoc networks (CRAHNs).

\section{Time series}

A time series can be defined as a set of quantitative observations $\left\{Y_{t}\right\}_{t \in \mathrm{T}}$ arranged in chronological order [29], indexed by an ordered set $T=\left\{t_{1}, t_{2}, t_{3}, \ldots, t_{n}\right\}$.

The time series analysis aims to: (1) Understand the underlying powers and structure that have formed the obtained data, and (2) Build a model and proceed to predicting future values of the observed phenomena. Time series analysis is used for many applications such as economic forecasting, sales forecasting, budgetary analysis, stock market analysis, process and quality control, etc. Recently, it starts being used in the field of computer networks communications. Indeed, time series have gained the attention of many researchers for the modeling of the Internet and wireless mobile networks traffic.

An important step while analyzing time series is to define the appropriate model corresponding to the observed data. A wide used approach to analyze time series is the ARMA (Auto Regressive Moving Average) analysis. An ARMA process has two components: Autoregressive component (AR) and Moving Average (MA) component. In an AR process, a random variable is explained by its past values rather than other variables. While in MA process, a random variable is explained by its actual mean, adjusted by a weighted sum of the errors that "corrupt" the previous values. ARMA analysis method was proposed by Box and Jenkins [29] and they have defined three steps to model and forecast time series:

1. Model Identification: this step is performed to identify the model structure using two main functions: the autocorrelation function (ACF) and the partial autocorrelation function (PACF).

2. Parameter Estimation: this step is performed to determine the coefficient of the linear combination of the identified model.

3. Forecasting: the ultimate goal is to forecast the future values of the time series based on the previous observed data and the linear combination determined at the second step. Therefore, ARMA ( $p$, q) model is defined as:

$$
y_{t}=c+\mu+\sum_{i=1}^{p} \varphi_{i} y_{t-i}+\sum_{j=1}^{q} \theta_{j} \varepsilon_{t-j}+e_{t}
$$

Where:

$p \quad$ Order of the process AR 
$q$ Order of the process MA

$\varphi_{i}$ Time-invariant coefficient of the AR model

$\theta_{i} \quad$ Time-invariant coefficient of the MA model

$e_{t} \quad$ Samples of white noise with mean zero and variance $\sigma^{2}$

$\varepsilon_{i} \quad$ White noise error terms

$c$ A constant

$\mu$ Expectation of $y$

$T$ Positive natural number

Box and Jenkins method requires that the time series must be stationary to be eligible for ARMA analysis. In order to check the stationarity, two conditions must be verified:

$$
\begin{aligned}
& E\left(y_{t}\right)=\mu \text { is constant independent of instant } t \\
& \operatorname{Cov}\left(y_{t}, y_{t-j}\right)=\gamma^{j} \text { only depends on time lag } j
\end{aligned}
$$

In the following section, we present in detail our proposed solution for video dissemination over vehicular networks ViCoV.

\section{PROposed ViCoV}

Video dissemination is a demanding task for any kind of network because of high bandwidth utilization and strict delay requirements. In VANETs, due to their intrinsically characteristics, such as the wireless medium and high dynamicity, this task becomes even harder. Therefore, the main goals of our proposed solution are to perform video dissemination in a reliable and efficient way without incurring a high load into the network. Toward these goals, ViCoV selects the best channel and a minimum set of vehicles to broadcast and determines also when the broadcast should take place and at what place. This way, the protocol tries to reduce the load sent to the link layer by decreasing the amount of redundant re-transmissions. Moreover, since the network partitioning is very common in these networks, received messages are kept in a local buffer to be later forwarded to uninformed vehicles.

In this section, we present our proposed solution for video streaming over cognitive radio VANETs (ViCoV). First, we present its general architecture, and then we go through the details of each of its components. $\mathrm{ViCoV}$ is modeled as an autonomic system. Each vehicle is considered as an autonomic element which selects the best channel / neighbor to broadcast the content according to the dynamic network changes. These changes concern the spectrum utilization, the network topology and connection. Towards these goals, the autonomic element (vehicle) implements the control loop presented in Figure 4.

$\mathrm{ViCoV}$ is composed of three mechanisms: The dynamic channel selection mechanism, the broadcaster selection mechanism and the store-carry-and-forward mechanism.

The dynamic channel selection mechanism selects the best channel for transmission. The channels are selected from the DSRC band or, if needed, a cognitive radio (non DSRC) channel is allocated. The Monitoring module senses the quality of the channel and PU activity on the channel, and calls the Analyze module which analyzes the quality of the channel and predicts the PU activity on the channel. Then, the Decision module selects the best channel for transmission based on the channel selection strategy stored in the knowledge base. 
The broadcaster selection module selects the best broadcasting neighbors in order to reduce redundant transmissions based on a new centrality metric that we proposed, while the store-carry-forward module is used by borders vehicles to keep content and send it later to uninformed vehicles.

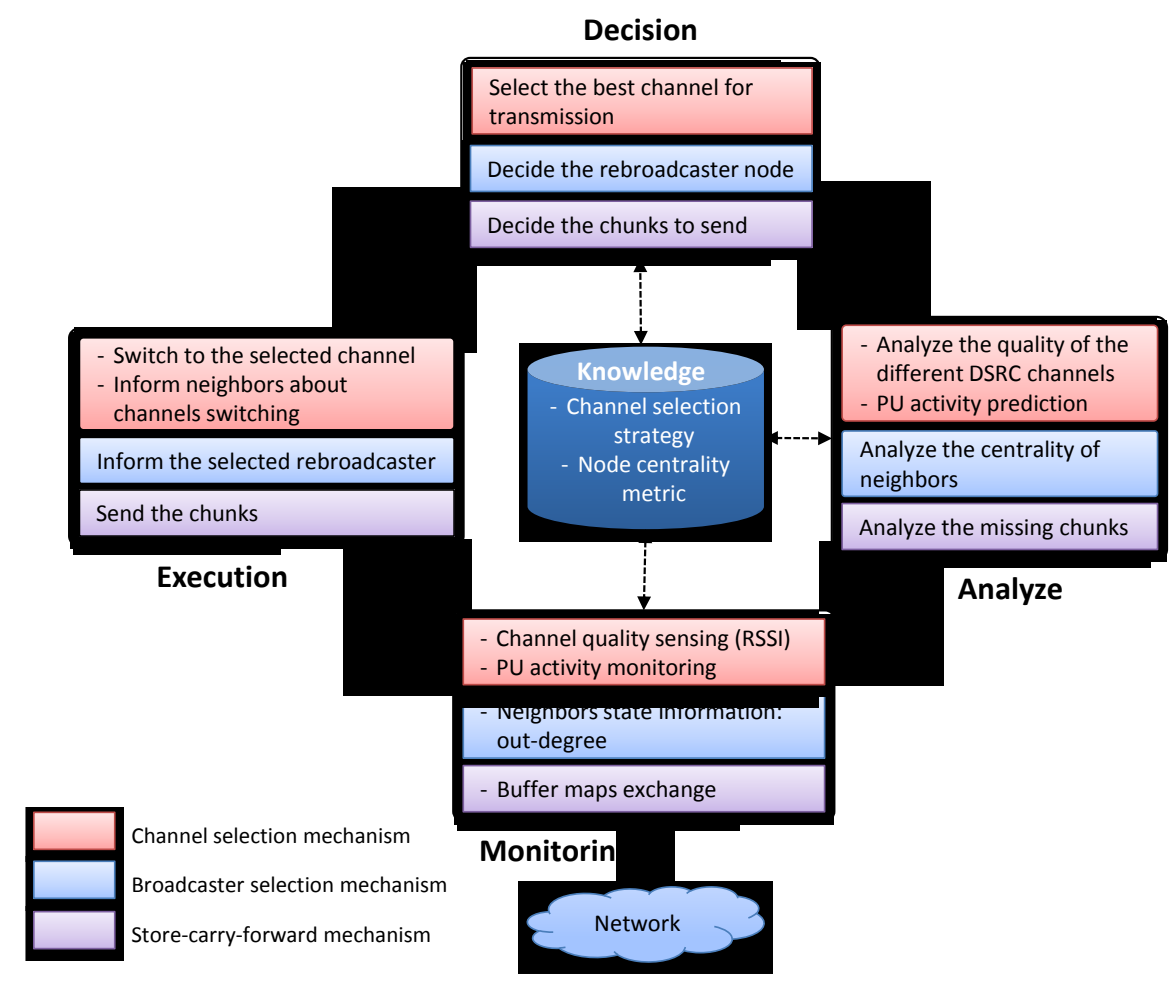

Figure 4: ViCoV architecture

\section{Dynamic channel selection}

In 1999, the FCC has assigned the DSRC band for vehicular communication; V2V and V2I communications. As shown in Figure 5, the DSRC band is subdivided into 7 channels: 1 control channel (Channel 178) and 6 service channels. Short range vehicular communication has been then standardized under the IEEE 802.11p Wireless Access in Vehicular Environments (WAVE) standard and further more in IEEE 1609.4 DSRC for multi-channel communication. Many studies [3] [30][31][32] show that neither the both mentioned standards, especially in dense scenarios, cannot ensure time critical message dissemination. In addition, video transmission using these standards and under high density conditions suffers from high packets loss ratio. Consequently, the quality of the received video degrades drastically. In our proposed solution, we use DSRC channels to transmit safety applications content in priority and then non-safety application content if free DSRC channels are still available. Otherwise, cognitive radio channels selected to transmit non-safety application content. In this case, the dynamic channel selection module selects the best CR channel which meets the QoS of the transmitted content and respects the PU priority.

In $\mathrm{ViCoV}$, we rely on two channels working simultaneously, i.e. OBUs are equipped with two transceivers: one transceiver tuned on the control channel $(\mathrm{CCH})$ and the other transceiver tuned on a service channel (SCH) (see Figure 6). The service channel could be a DSRC channel (except channel 178) or a CR channel. It is used for content transmission, while the control channel is used to exchange beacons, topology information, channels negotiation and synchronization messages. 


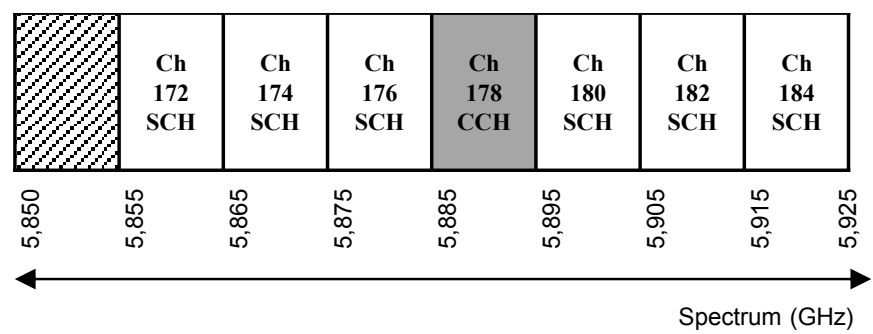

Figure 5: DSRC band plan channel designations

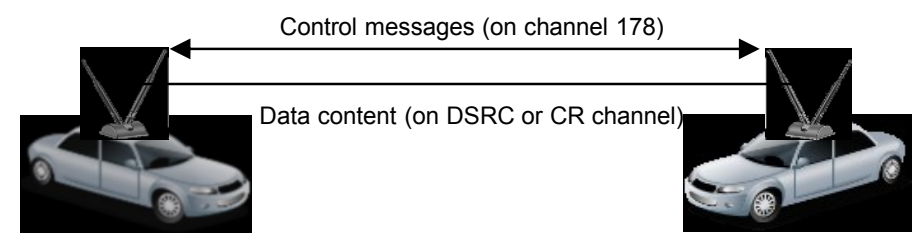

Figure 6: Communication mode in ViCoV (Two transceivers' OBU)

In the following we detail the channel selection mechanism. We first present the exchanged messages and their structure. Then, we go through the channel selection protocol functionalities, detailing the channel negotiation protocol and the cognitive radio channels selection mechanism.

\subsection{Data structure}

Dynamic channel selection module relies on special signaling messages to perform negotiations between vehicles. These messages are exchanged via the control channel. Their main task is to establish the communication between the sender and receiver vehicles on the service channel.

- Beacon Messages: (Figure 7-a) classic vehicle beacon messages sent periodically by vehicle to announce its ID, speed, position and direction. In addition to a Boolean field "Rebroadcaster" which indicates if the vehicle is selected as a rebroadcaster node. Only rebroadcaster vehicles are allowed to retransmit the content.

- Chanel state announcement: (Figure 7-b) message broadcasted by a sender vehicle to announce new transmission. This message contains the transmission channel and the priority level of the content. In ViCoV, we define 4 content priority levels: safe / non-safe content and real-time / nonreal time QoS requirement content. The content priority levels are presented in Figure 9 with some practical examples.

- Request to switch: (Figure 7-c) message sent by a sender vehicle, having high priority content to send, to vehicle transmitting low priority content (non-safe content) on DSRC channel. The destination vehicle of this message is requested to release the channel and switch to CR channel.

- Clear to switch: (Figure 7-d) message sent in answer to Request to switch message to inform the sender vehicle that its request is received, the switch process is initiating and the requested channel is now free.

- Channel reservation: (Figure 7-e) message sent by a vehicle to reserve a channel for incoming transmission. The content priority level is specified in this message.

- Channel reservation approved: (Figure 7-f) message sent in response to Channel reservation message. It announces to the requester vehicle that his application is approved and the reserved channel is free. 
Channels occupation table (COT) (Figure 8): Each vehicle in the network maintains and updates the neighbors' channels in the channels occupation table (COT). This table is updated at the reception channel state announcement messages. The COT table stores the following information for each inuse channel:

- Channel: The frequency channel.

- Channel's user vehicle ID: The ID of the vehicle using the channel.

- Current content priority level: The priority level of the transmitted content.

- Reservation - vehicle-ID: The ID of the vehicle reserving the channel for an incoming transmission.

- Reservation - Content priority level: The priority level of the content to be transmitted by the requester vehicle.

In the following sub-section we present the detail of the channel selection mechanism which makes use of the presented messages in this sub-section.

\begin{tabular}{|l|l|l|l|l|}
\hline ID & Speed & Position & Direction & Rebroadcaster \\
\hline
\end{tabular}

a) Beacon Messages

\begin{tabular}{|c|c|c|}
\hline $\begin{array}{c}\text { Selected channel to } \\
\text { switch to }\end{array}$ & Content priority level & $\begin{array}{c}\text { Receiver } \\
\text { ID }\end{array}$ \\
\hline
\end{tabular}

c) Request to switch

\begin{tabular}{|l|l|l|}
\hline Channel & Content priority level & Requester ID \\
\hline
\end{tabular}

e) Channel reservation

\begin{tabular}{|l|l|}
\hline Channel & Content priority level \\
\hline
\end{tabular}

b) Chanel state announcement

\begin{tabular}{|l|l|}
\hline Agreed switch-to channel & Requester ID \\
\hline
\end{tabular}

d) Clear to switch

\begin{tabular}{|c|l|}
\hline Channel & Requester ID \\
\hline
\end{tabular}

f) Channel reservation approved

Figure 7: Control messages structure

\begin{tabular}{|c|c|c|c|c|}
\hline Channel & $\begin{array}{c}\text { Channel's user } \\
\text { vehicle ID }\end{array}$ & $\begin{array}{c}\text { Current content } \\
\text { priority level }\end{array}$ & $\begin{array}{c}\text { Reservation- } \\
\text { vehicle-ID }\end{array}$ & $\begin{array}{c}\text { Reservation-Content } \\
\text { priority level }\end{array}$ \\
\hline
\end{tabular}

Figure 8: Channels occupation table (COT)

\begin{tabular}{|c|l|l|}
\hline Content priority & Content & \multicolumn{1}{c|}{ examples } \\
\hline 1 & Safety - real time & Real time video of accident scene \\
\hline 2 & Safety - non real time & $\begin{array}{l}\text { Emergency message: accident, Oil } \\
\text { Stain, Icy Road ... }\end{array}$ \\
\hline 3 & Non safety - real time & Advertising video, films \\
\hline 4 & Non safety - non real time & e-mails, web surfing \\
\hline
\end{tabular}

Figure 9: Content priority levels

\subsection{Dynamic channel selection mechanism}

The dynamic channel selection mechanism selects the best available channel in terms of noise / interferences and channel stability regarding the PU activity, while giving preemptive priority to safety content. The flowchart of the proposed mechanism is presented in Figure 10. 


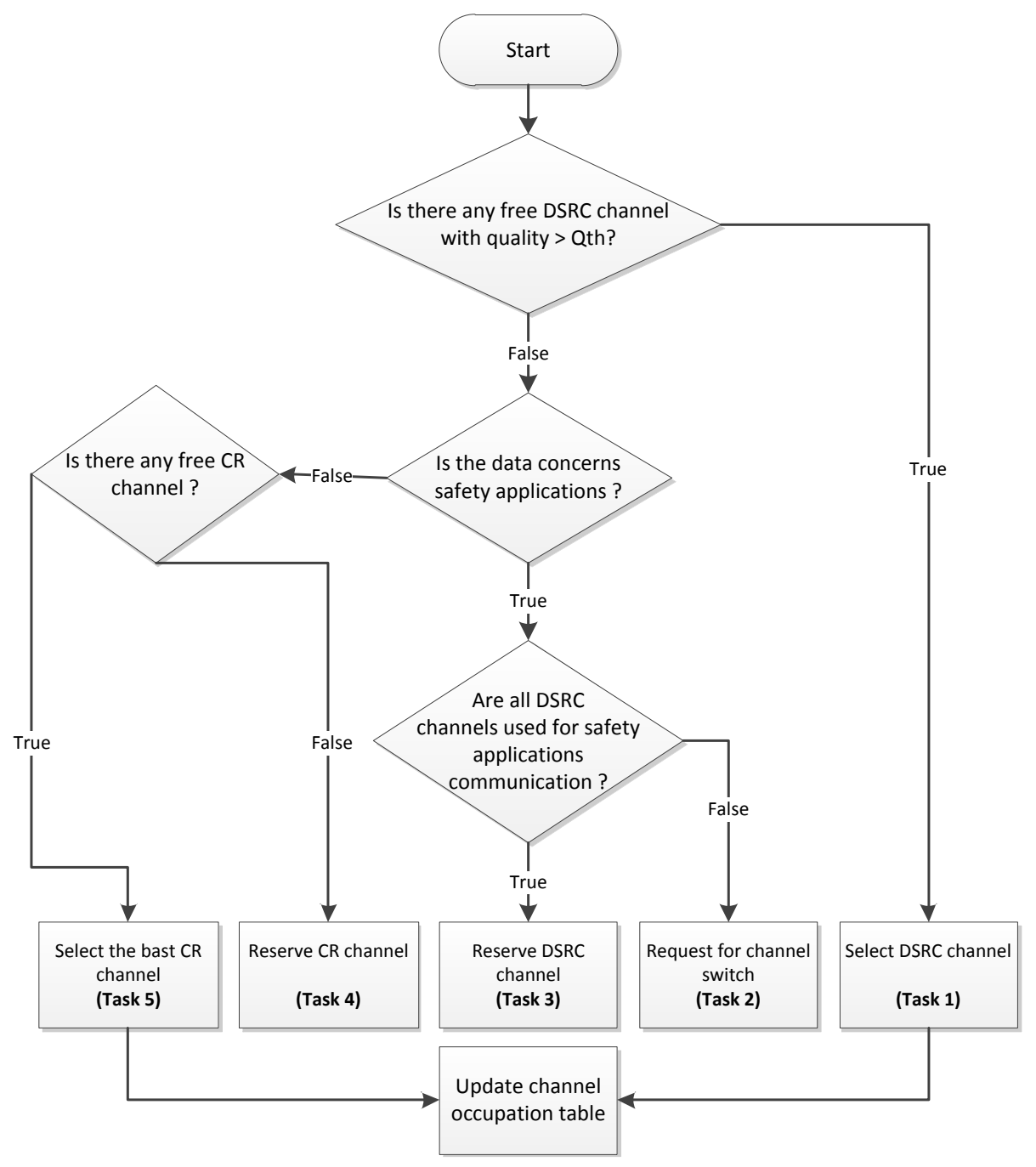

Figure 10: Dynamic channel allocation protocol flowchart

In order to measure the channel quality, we adopt the Received Signal Strength Indicator (RSSI) metric which is approved to be appropriate for wireless networks [34]. Its values range from 0 for worst signal quality to 30 for the best signal quality.

A vehicle with a content to transmit looks in its COT table for a free DSRC channel and checks its quality level. A channel is considered of a good quality if its RSSI is higher than a certain threshold $\mathrm{Q}_{\text {th }}$ (in our simulation $\mathrm{Q}_{\text {th }}=15$ ). If a good quality free channel is available, the sender vehicle selects the channel, broadcasts Channel state announcement message on the control channel and starts transmitting content.

If no DSRC channel is available, or none of them meets the required threshold quality, two cases should be considered:

- The content to be sent is a safety content: in this case, the COT table should be checked to verify if there are DSRC channels transmitting non safety content. If it is the case, the vehicle requisitions the channel having lowest content priority level, and sends Request to switch message to the concerned vehicle. Otherwise, if all DSRC channels are used for safety content, the vehicle broadcasts a Channel reservation message. 
- The content to be sent is non safety application content: in this case, the vehicle looks for a free CR channel. We mean by CR channel all radio channels except the DSRC channels. Some of these channels are licensed, and in this case, the channel can be used with respect to the PU priority. ViCoV selects the best available CR channel in terms of RSSI and stability over the time. To estimate the stability of CR channel, we developed PUs activity forecast module which predicts the PU activity based on time series model. This module is detailed in section 1.4. In the case where there is no available CR channel, the requester vehicle reserves the CR channel that will be released first and broadcast a Channel reservation message.

We note that the safety application messages are always sent over a DSRC channel using the $802.11 \mathrm{p}$ standard. The main reason behind this strategy is the fact that the standard handles many issues of the vehicular communications, mostly the security and the retransmissions. In order to ensure timely and secure delivery of the safety application messages and to be compliant with standard, we proposed that the safety message should be always sent via DSRC channels. However, non-safety application content can be sent over the CR channel if no DSRC channel is available.

In the following sub-section, we present in detail the channel negotiation protocol between a sender vehicle and the receivers.

\subsection{Channel negotiation protocol}

A sender vehicle after executing the dynamic channel selection mechanism (presented in 1.2) is left with five cases:

- Free DSRC / CR channel selection (Figure 10- task (1)): in this case, the sender vehicle broadcasts Channel state announcement message on the control channel and starts transmitting content on the service channel $(\mathrm{SCH})$. Receiver' vehicle checks the priority level of the content. If it is already receiving a less priority content on a different channel, it stops reception on that channel and switch to the new announced channel. Here we note that the content priority levels that we defined in Figure 9 can be adjusted to the end user preferences. For instance, user can prefer to stay surfing on the web even there is a real time content available (film) announced in new channel. In this case, "Non safety real time" and "Non safety - non real time" content should have the same priority level.

- Request for channel switch (Figure 10-task (2)): In this case, the vehicle sends a Request to switch message. The destination vehicle verifies that the content to be sent is of more priority than the content it is transmitting. If it is the case, it answers with Clear to switch message and selects a CR channel to transmit its content. The $\mathrm{CR}$ channel is selected using the $\mathrm{CR}$ channel selection module defined in 1.4. A new Channel state announcement message is sent and the task (1) is then executed by the requester vehicle.

- DSRC channel reservation (Figure 10-task (3)): a channel reservation message is sent to the current channel user. The destination vehicle updates its COT table, by positioning the fields "Reservation-ID vehicle" and "Reservation-Content priority level". This latter field helps to prioritize the reservation requests. Once, the channel user finishes transmission its content, it selects the requester vehicle with highest content priority and broadcasts a Channel reservation approved message. The selected vehicle broadcasts Channel state announcement message and starts transmission. 
- CR channel reservation (Figure 10-task (4)): if all CR channels are used, the requester vehicle selects, using the module described in 1.4, the best $\mathrm{CR}$ channel and broadcasts a channel reservation to inform its neighbors that this channel is reserved for incoming transmission.

- CR channel selection (Figure 10- task (5)): If CR channels are available, ViCoV selects the most stable one by estimating the PU activity in each available CR channel. The CR channel selection mechanism is presented in detail in the following sub-section.

\subsection{Cognitive radio channel selection}

In this section we detail the cognitive radio channel selection module, which selects the best available CR channel by measuring the channels RSSI and estimating the PU activity. A first selection of the available channels is done by eliminating channels with RSSI bellow the quality threshold $\mathrm{Q}_{\text {th }}$. Then, the channels are classified based on the estimation of PU activity on each channel. Indeed, more the channel is stable (no PU activity on the channel) more the SU is not required to switch from a channel to another. This switch operation between channels introduces a certain delay which is due to the fact that the physical transceiver switch from a channel to another one is not instantaneous. Thus, a CR channel with low PU activity should be promoted. In the following we detail the PU activity estimation mechanism using time series model.

First, we note that the physical detection of PU channel occupancy is done by a physical layer module which indicates at time $t_{i}$ if PU is actually using the channel or it is in idle mode [34].

In order to study the evolution of PU activity over time we define random variables $x_{t}, y_{t}$ as the time duration over which the $\mathrm{PU}$ is active, inactive on a channel $f$. Based on the consecutive values of $x_{t}$ and $y_{t}$, we construct the times series $\left\{x_{t}\right\}_{t \in N},\left\{y_{t}\right\}_{t \in N}$ where $x_{i}$ denotes the duration of the $i^{\text {th }} \mathrm{PU}$ activity period and $y_{i}$ denotes the $i^{\text {th }} \mathrm{PU}$ inactivity period. $\mathrm{N}$ is the set of naturel integers.

In our analysis, we use the data set resulting of the spectrum measurement study performed by RWTH Aachen University [36]. The measurements, collected from December $27^{\text {th }}, 2006$ to January $2^{\text {nd }}, 2007$ concern the $20 \mathrm{MHz}$ to $6 \mathrm{GHz}$ bands, where most of wireless services work today. We applied the Boxand-Jenkins [29] method to analyze time series. We should, before, to verify the stationarity of the two time series $\left\{x_{t}\right\}_{t \in N}$ and $\left\{y_{t}\right\}_{t \in N}$. Thus, we run the stationarity test:

$$
\begin{gathered}
E\left(z_{t}\right)=\mu \text { Where } \mu \text { is constant independent of instant } t \\
\operatorname{Cov}\left(z_{t}, z_{t-j}\right)=\gamma^{j} \text { Only depends on time lag } j
\end{gathered}
$$

Where $\left\{z_{t}\right\}_{t \in N}$ represents the studied time series $\left\{x_{t}\right\}_{t \in N}$ or $\left\{y_{t}\right\}_{t \in N}$.

The obtained results showed that the two stationarity conditions are satisfied in all the time series resulting from the data. Consequently $x_{t}$ and $y_{t}$ can be analyzed using Box and Jenkins method. For lack of space, we describe in this section the Box and Jenkins steps for the times $\operatorname{series}\left\{x_{t}\right\}_{t \in N}$. The same process is applied to the series $\left\{y_{t}\right\}_{t \in N}$ to determine its model parameters. We dubbed this model as "ViCoV PU occupancy model"

\section{Step 1: ViCoV PU occupancy model}

In order to analyze the resulting time series, we have used Minitab [37]. Figure 11 and Figure 12 show the autocorrelation function (ACF) and the partial autocorrelation function (PACF) of $\left\{x_{t}\right\}_{t \in N}$, respectively. The results indicate that the best appropriate model is the $\operatorname{ARMA}(3,1)$, since PACF 
presents three important peaks (this approves the AR(3) part), and the ACF presents one important peak (this approves the MA(1) part).

Based on the obtained results, the ViCoV PU occupancy model can be written as:

$$
x_{i}=\mu+\varphi_{1} x_{i-1}+\varphi_{2} x_{i-2}+\varphi_{3} x_{i-3}+\theta_{1} \varepsilon_{i-1}+\varepsilon_{i}
$$

Where $\mu$ represents the mean of $x_{i}$.

$\varphi_{1}, \varphi_{2}, \varphi_{3}$ and $\theta_{1}$ denote the ViCoV PU occupancy model parameters $\left(\varphi_{1}, \varphi_{2}, \varphi_{3}\right.$ related to the AR part and $\theta_{1}$ related to the MA part).

$\varepsilon_{i}$ and $\varepsilon_{i-1}$ are supposed to be independent, identically distributed random variables derived from a normal distribution with zero mean $\varepsilon_{i} \sim \mathrm{N}\left(0, \sigma^{2}\right)$ where $\sigma^{2}$ is the variance.

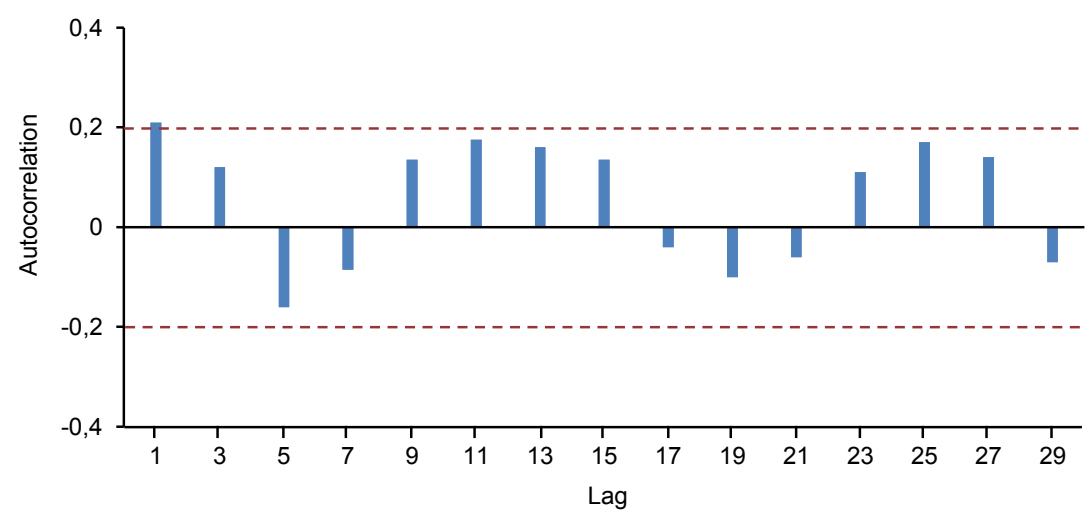

Figure 11: Autocorrelation Function (ACF) plot for PU activity duration

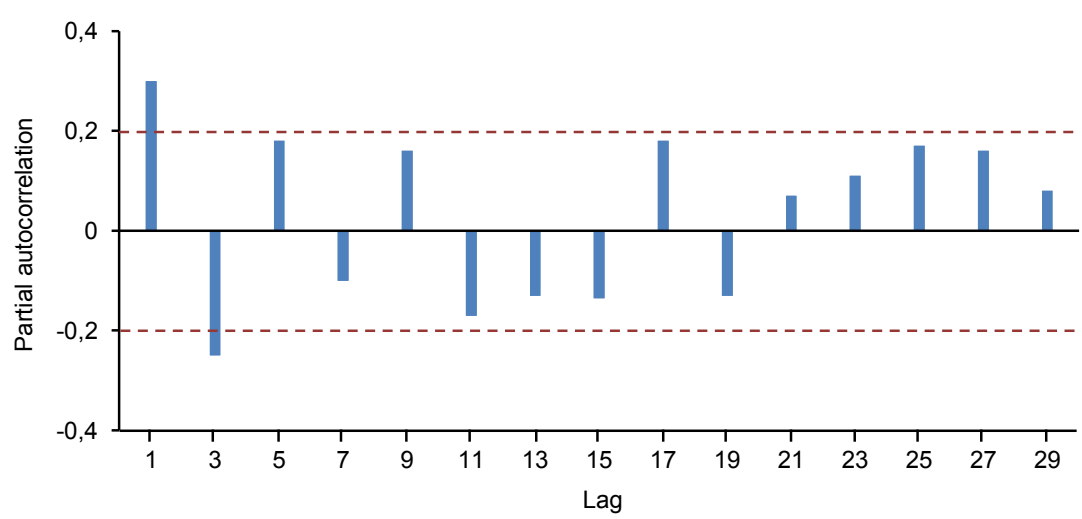

Figure 12: Partial Autocorrelation Function (PACF) plot for PU activity duration

We applied the same test to the PU inactivity time $\left\{y_{t}\right\}_{t \in N}$ and we conclude that it follows an ARMA $(3,1)$ model.

\section{Step 2: ViCoV PU occupancy model Parameters Estimation}

After identifying the order of the ViCoV PU occupancy model, the next step is to estimate its parameters. For the autoregressive part (AR), the parameters can be computed using the Yule-Walker equations [38]. The basic idea behind the Yule-Walker equation relies on the fact that there is a direct relation between the parameters $\left(\varphi_{i}, i=1 \ldots p\right)$ and the covariance function of the times series. This direct relation can be reversed to define the parameters from the ACF which give the Yule-Walker equations: 


$$
\gamma_{m}=\sum_{k=1}^{p} \varphi_{k} \gamma_{m-k}+\sigma_{\varepsilon}^{2} \delta_{m}
$$

Where $\mathrm{m}=1, \ldots, p$ yielding $(p+1)$ equations. $\gamma_{m}$ is the auto-correlation of $\mathrm{Y}, \sigma_{\varepsilon}$ is the standarddeviation of the input noise process, and the $\delta_{m}$ is the Kronecker Delta function. Using Equation (8) AR (p) parameters can be estimated by replacing the covariance with its estimated values. The estimated AR parameters are then used to deduct the MA parameter by identification.

\section{Step 3: Forecasting}

Once the ARMA model identified and its parameters computed, a CR node can predict the PU activity on the channel. In practice many simplified and efficient methods have been proposed for computing the ARMA model parameters. In ViCoV we adopt the online ARMA parameters estimation proposed in [39], where authors propose to compute these parameters in an incremental fashion. For each available channel, the $\mathrm{CR}$ computes the next PU activity date $\left(A_{f}\right)$ on a channel $f$ as follows:

$$
A_{f}=\left|\sum_{i=0}^{i=k} y_{i}\right|
$$

Where:

$t:$ the current time.

$k$ : the activity period or inactivity period that covers the current time $t$. Hence, the sum $\sum_{i=0}^{i=k} y_{i}$ represents the sum of the activity and inactivity periods from $\mathrm{t}=0$ until the next PU inactivity period, which is the date of the next PU activity.

As practice utilization scenario, the ARMA model and its parameters can be dynamically computed and updated by a central authority in each city and provided as a service to vehicles via a road side unit and propagated in the vehicles network in ad-hoc manner as a safe message. The vehicle having the ARMA model and its parameters forecast the PU activity on each available channel and select the channel with long stability and an acceptable RSSI.

In this section we presented the dynamic channels selection component of ViCoV. First, we presented the data structure of the exchanged messages. Then, we provided a global overview of the channels selection mechanism. After that, we detailed the channels negotiation protocol, and finally we detailed the CR channel selection mechanism. In the following we present the second component of $\mathrm{ViCoV}$ (Figure 4) namely the broadcaster selection mechanism.

\section{Rebroadcaster selection mechanism}

In a dense environment, where the spectrum availability is more and more rare, we presented in the previous section the first module of $\mathrm{ViCoV}$ a mechanism to dynamically select the best channel for transmission. This scheme can be enhanced by eliminating the useless redundant retransmissions. Indeed, the traditional broadcasting mechanism advocates that each node in the network receiving the content will rebroadcast it. This intensive rebroadcasts increase the interferences. Consequently, the packets loss increases and the video quality is degraded. This funding prompted us to enhance our video streaming system over VANET by the Broadcaster selection mechanism, which selects a minimum subset of neighbors' vehicles to rebroadcast the content. 
The proposed mechanism is inspired from the Social Network Analysis (SNA) methods to select the central nodes in their communities. Indeed, the rebroadcaster nodes should be as central as possible in the network in order to broadcast the content to a maximum number of neighbors without need to further retransmissions. The problem of broadcaster's nodes selection is illustrated in Figure 13, where a roadside unit is broadcasting content in a road corner. Only vehicles A, B, C are in its range. The other vehicles receive the content in ad-hoc manner. In this example only nodes A and C (and not B) are selected to rebroadcast the content. Then only node $\mathrm{G}$ (and not H or I) will rebroadcast again the content. In this case, 3 redundant rebroadcasts are omitted, namely the rebroadcast of nodes $\mathrm{B}, \mathrm{H}$ and I.

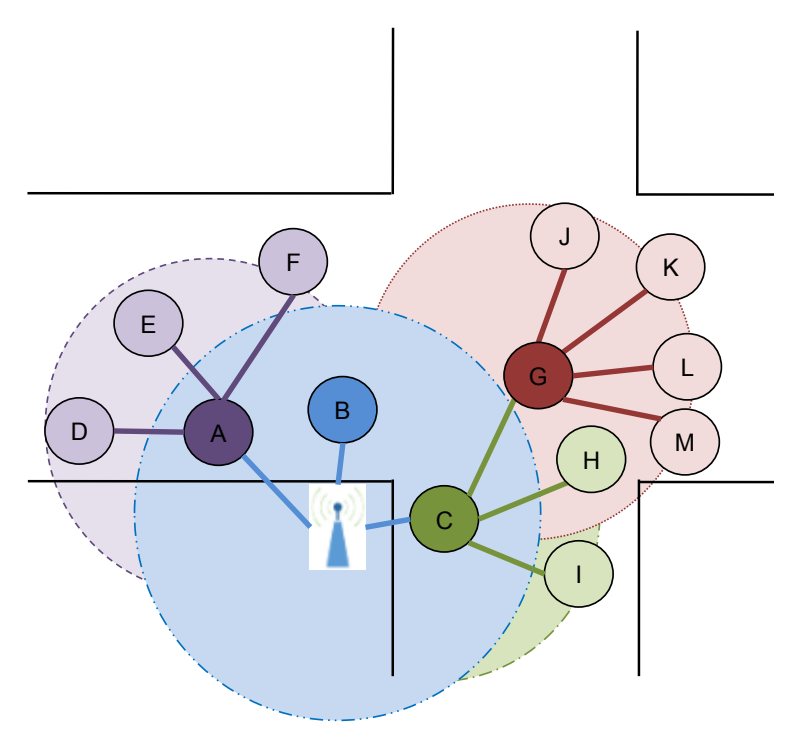

Figure 13: Example of rebroadcaster nodes selection

In ViCoV, we select a sub set of neighbors' vehicles, which ensure a large dissemination in the network, to rebroadcast the content. Our target is then, to select a minimum set of neighbors' vehicles which are more "central" in the network and which covers all the 2 hops neighbors. In Social Network Analysis (SNA) the centrality problem has been widely studied [40] and many centrality metrics have been proposed such as the node out-degree, the Shortest-Path Betweenness Centrality (SPBC) [32]. In the example of Figure 14, we note that the nodes C, D, F and G are equally central in terms of outdegree; they have all an out-degree $c$ equal to 4. In addition, if we calculate the SPBC [40] for each node in the graph, we found that node $\mathrm{G}$ is the most central $(\mathrm{SPBC}=13)$, followed by nodes $\mathrm{C}, \mathrm{D}$ $(\mathrm{SPBC}=10,8$ respectively) and finally node $\mathrm{F}(\mathrm{SPBC}=7)$. This is somewhat unexpectedly, since node $\mathrm{F}$ has all network nodes at its range (at distance 2-hops). Based on this observation, we propose a new centrality metric, named the dissemination capacity (DC) defined as follows:

Definition: The dissemination capacity $\operatorname{DC}(v)$ is the maximum degree $n$ of the node $v$, which ensures that $n$ of its 1-hope neighbors has a degree equal or greater than $n$.

Applying this definition to the graph in Figure 14, we find that $\mathrm{DC}(\mathrm{G})=\mathrm{DC}(\mathrm{D})=2$, whereas $\mathrm{DC}(\mathrm{F})=$ $\mathrm{DC}(\mathrm{C})=3$. 


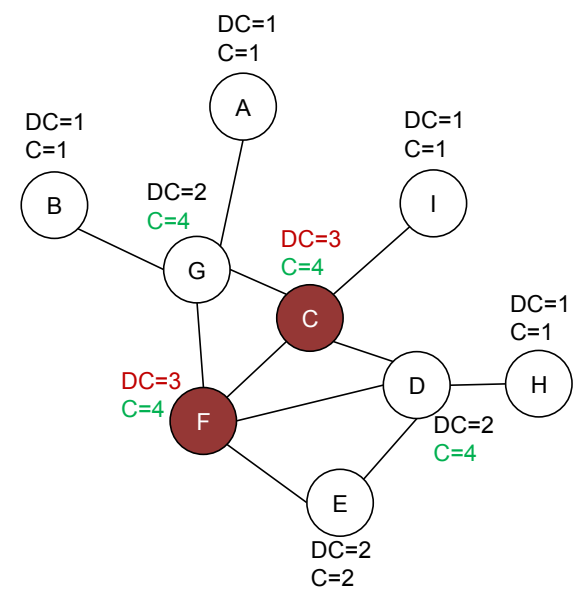

Figure 14: Node dissemination capacity example

It is clear that the network nodes which have more connections (larger degree) are more likely to be "powerful" to disseminate the content in the network, since they can directly touch more other nodes. However, their power depends also on the degrees of their 1-hop neighbors. Large values for the $\mathrm{DC}(v)$ of a node $v$ indicate that this node $v$ can reach others nodes on relatively short paths. Consequently the proposed centrality metrics, in addition to the reducing the amount of redundant rebroadcast, allows also to minimize the end to end delay.

Despite of the advantages that the DC metric offers, its calculation is not computational costly neither introduces an important communication overhead. Indeed, each vehicle in the network computes locally its DC and disseminates it to its neighbors in beacon message. In order to compute its DC, a vehicle needs only the out-degree of its 1 -hope neighbors. This parameter is piggybacked in beacon message broadcasted periodically. The refined structure of beacon message is provided in Figure 15.

\begin{tabular}{|l|l|l|l|l|l|l|l|}
\hline ID & Speed & position & direction & Rebroadcaster & Out-degree & Dissemination capacity (DC) & Buffer map \\
\hline
\end{tabular}

Figure 15: Refined structure of Beacon message

In this sub-section, we presented the rebroadcasters selection mechanism which aims to reduce interferences in high densities environments. In low densities environment, ViCoV relies on the Storecarry-forward mechanism which is presented in the following sub-section.

\section{Store-carry-forward mechanism}

Even though $\mathrm{ViCoV}$ is intended to guarantee high content delivery by avoiding channel overloading, some message losses may still happen due to the demanding nature of video dissemination. Furthermore, as shown in [41][42], VANETs are naturally intermittently connected networks. This means that even if the channel is not overloaded and there are no messages collisions, $100 \%$ content delivery cannot be guaranteed due to the lack of a lasting end-to-end path from source to intended recipients. With this in mind, we propose a store-carry-forward mechanism to increase the content delivery capability of $\mathrm{ViCoV}$ by serving vehicles that failed to receive messages from the initial dissemination process due to the intermittent disconnection of the network.

In $\mathrm{ViCoV}$, a vehicle receiving a message for the first time, stores it in a local buffer until the message's time-to-live expires or the vehicle leaves the region of interest of the message. Notice that, both parameters are specified by the application that generated the message. For instance, the time-to- 
live for a message may be 2 minutes long and the region of interest may comprise all vehicles in a 1 $\mathrm{km}^{2}$ area around the source.

Besides storing the message, the vehicle needs to notify its neighbors about all the messages it has received so far that they are still in its local buffer. This way, if a neighbor finds out that the vehicle has not received some messages, it will forward them. To accomplish that, the IDs of all stored messages are piggybacked in the periodic beacons exchanged among vehicles. For instance, if a vehicle A has received messages with IDs 1 and 2, it inserts these IDs in the beacons to act as a buffer map. Therefore, if a neighbor of A, say B, has received messages with IDs 1,2 and 3, when it receives the beacon from A and notices the missing ID 3, it will forward this message to A.

In $\mathrm{ViCoV}$, the store-carry-forward mechanism is used not just for sparse traffic scenarios, but also for high traffic conditions. In this case, coordination between the neighbors' vehicles is needed before retransmission. For instance, imagine that the sender is the only vehicle that has not received a given message. Therefore, when it transmits a beacon that does not acknowledge the receipt of this message, all its neighbors will attempt to forward the message, probably leading to message collisions, contention and waste of network bandwidth. To overcome such issue, in Store-carry-forward mechanism, the priority to broadcast is given to the designed rebroadcaster vehicles if it exists in the neighborhood. Indeed, in the forward mechanism that we propose (see Figure 16), when a vehicle received a beacon message, if it is a rebroadcaster vehicle, it rebroadcasts the entire missing messages announced in the beacon. If is not a rebroadcaster, it checks if there is a rebroadcaster vehicle in its neighborhood which can serve the vehicle sending the beacon. If it is not the case, the vehicle rebroadcasts the missing messages. In this manner a redundant rebroadcasts are avoided.

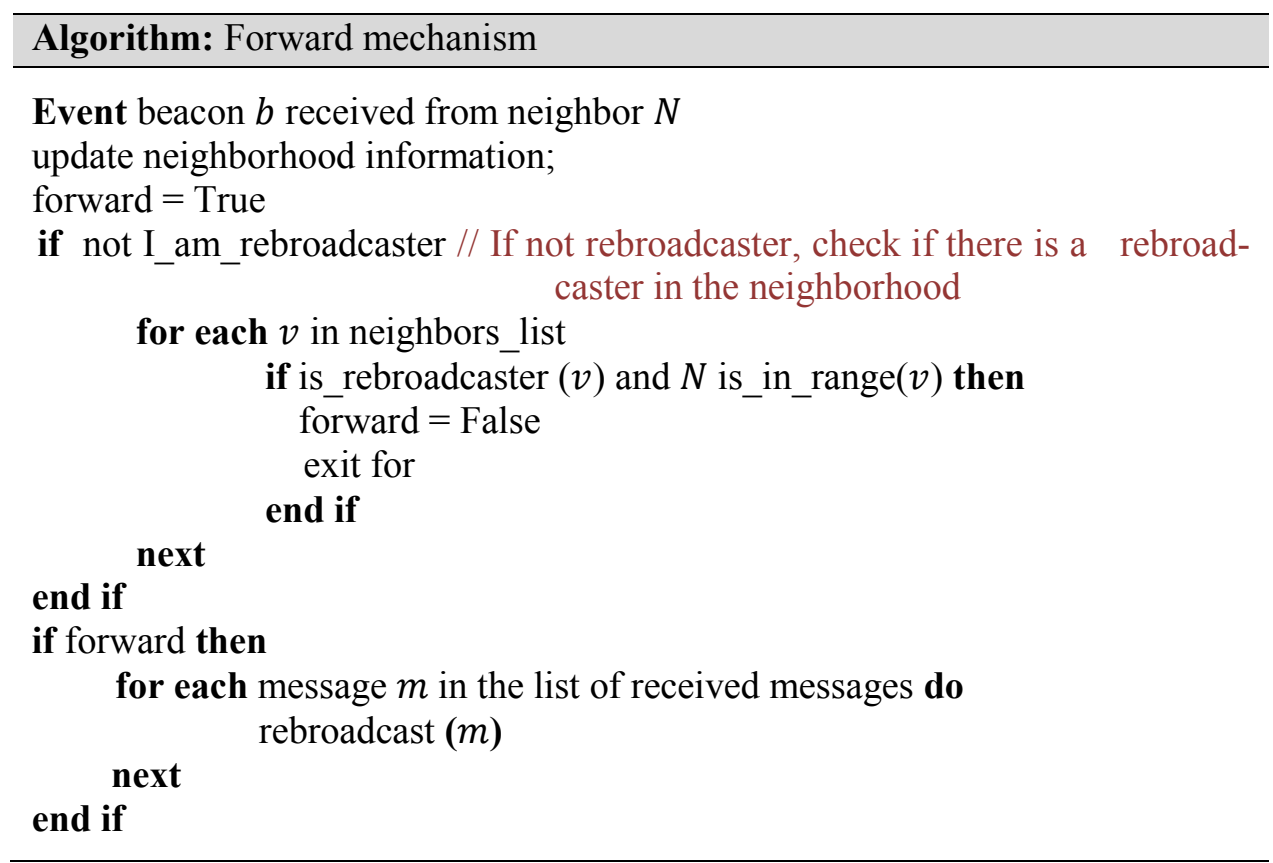

Figure 16: Forward mechanism

\section{Procesing overhead}

The buildup process of the ARMA model adopted in ViCOV may take relatively high memory and computational overhead. However, the availability of high speed computing overcomes this problem and allows processing the huge dataset for ARMA to build a statistical model [32], especially when, like in our solution, the process is done offline. Indeed, in ViCOV, the model parameters and coefficient are computed and distributed via RSUs to vehicles as a value-added service. The vehicle 
just makes use of these parameters to forecast the PU activity on each channel and select the most stable one.

In addition, in ViCOV, the exchanged messages are lightweight, and efficiently codded. For instance the buffer map is binary coded: 1 to represent the chunk presence and 0 to represent its absence. This reduces the size of the hello messages exchanged periodically between vehicles to announce their presence and their content.

Finally, the intelligent cognitive radio channels selection, which aims to reduce the channel switching, optimizes the number of exchanged control messages, such as the Request to switch, Clear to switch, Channel reservation, Channel reservation approved, by reducing the number of channels switch. This reduces also the overhead in our solution.

\section{PERFORMANCE ANALYSIS}

In this section we evaluate the performance of our proposed mechanism $\mathrm{ViCoV}$, using the network simulator NS-2 coupled with SUMO to generate real traffic mobility. Evaluation metrics were captured and compared to the multichannel vehicular communication standard IEEE 1609.4 [32] and the protocols UV-CAST [16] and AID [11]. Recall from our discussions on section II that AID and IEEE 1609.4 were designed for always-connected networks. However, UV-CAST, like our solution, was designed to work under varying traffic conditions, since it implements both broadcast suppression and store-carry-forward mechanisms. The performance of the four protocols are compared in terms of frame loss, Peak Signal-to-Noise Ratio (PSNR), frames delay and total number of messages transmitted [46].

\section{Simulation environment}

To evaluate our proposed protocol, the implementation and simulation were performed using NS-2 version 2.31 [44] and we used SUMO [45] for traffic generation. SUMO performs simulations of vehicle movements in real word maps following multiple lanes, speed limits and traffic lights. Different maps of $4 \mathrm{Km} \times 3 \mathrm{Km}$ from Aachen city were selected using the OpenStreetMap Project [43]. The map data includes all roads attributes such as driving direction, stop signs, speed limits, lanes count and road type. Vehicle routes are computed using the DUAROUTER [45]. Routes computation takes into consideration the street length, speed limits, lane count, and street type to select the shortest route. In addition, in order to increase the accuracy of our simulation, different building are added using the POLYCONVERT [45] application in SUMO.

For the vehicle behavior model, we adopted in our simulation the CarFollowing Model developed by Krauss which is based on the following principle: the drive tries to stay away from the vehicle in front of him at a distance and velocity that allow him to react properly to the other vehicle behavior. The model is based on the following parameters: Maximum vehicle speed, vehicle length, vehicle acceleration, vehicle deceleration, and driver imperfection. The values of these parameters in our simulation are provided in Table 1.

\section{Simulation parameters}

The radio propagation model adopted in our simulation is two-ray ground implemented in NS2. If not specified otherwise, the vehicle transmission range is $300 \mathrm{~m}$. In $\mathrm{ViCoV}$, Beacon messages are transmitted every $1 \mathrm{~s}$ on the DSRC control channel. 
Regarding the transmitted video, we adopt the widely used akiyo cif video, composed of 300 frames at a resolution of $360 \times 486$. The frames are packed up in 560 messages of 1024 bytes. The video is initially transmitted by a vehicle at the center of the network. In addition, the video blocks are generated at different bitrates: $100 \mathrm{kbps}, 500 \mathrm{kbps}$ and $1 \mathrm{Mbps}$. In order to evaluate the PSNR metric of the received video we use the video quality evaluation tool-set EvalVid [47] on the reconstructed raw videos.

We note that the obtained results are the mean of 30 excursions for each scenario represented at a confidence interval of $95 \%$.

\begin{tabular}{ll}
\hline Parameter & Value \\
\hline Vehicle Acceleration & $2.5 \mathrm{~m} / \mathrm{s}^{2}$ \\
Vehicle Deceleration & $4.6 \mathrm{~m} / \mathrm{s}^{2}$ \\
Vehicle Length average & $5 \mathrm{~m}$ \\
Maximum Vehicle Speed & Varying depending on simulation \\
& (from $1 \mathrm{~km} / \mathrm{h}$ to $140 \mathrm{~km} / \mathrm{h})$ \\
Driver Imperfection & 0.5 \\
\hline
\end{tabular}

Table 1: SUMO vehicle parameters

\section{Results}

\subsection{Frame loss}

In Figure 17, we study the frame loss in ViCoV, UV-CAST, IEEE1609.4 and AID while varying the vehicle densities from 20 to 400 vehicles/ $\mathrm{Km}^{2}$ under different video bitrates conditions: $100 \mathrm{kbps}, 200$ kbps and $1 \mathrm{Mbps}$.

Figure 17 (a) presents frame loss in the four protocols at $100 \mathrm{kbps}$. The common remark in this scenario is that in low vehicle densities scenarios $\left(<150\right.$ vehicles $\left./ \mathrm{km}^{2}\right)$, high frame losses is observed for all protocols due to the intermittently connection of the network. Indeed, when the network is intermittently connected, the connections between vehicles are shorts and at lower bitrates it takes longer time to stream the whole video from a vehicle to another. This leads to high frames loss. However, the frames loss is lower in ViCoV and UV-CAST thanks to the store-carry-forward mechanism that they implement.

In high vehicle densities ( $>150$ vehicles $/ \mathrm{km}^{2}$ ), we note that ViCoV and UV-Cast presents low frame loss thanks to the rebroadcast suppression mechanism and the channel/rebroadcasters selection mechanism implemented by ViCoV and UV-Cast, respectively. Here, we note lower frame loss in $\mathrm{ViCoV}$ comparing to UV-Cast, which shows the effectiveness of $\mathrm{ViCoV}$ channel and rebroadcaster selection mechanism.

Increasing the bitrate to $500 \mathrm{kbps}$ (Figure 17 (b)), the channel turns out to be overloaded and the advantages of $\mathrm{ViCoV}$ becomes more visible. Indeed, we note that the frame loss in ViCoV is almost zero at densities higher than 200 vehicles $/ \mathrm{km}^{2}$, while it is about $13 \%$ in UV-CAST, $23 \%$ in IEEE 1609.4 and $40 \%$ in AID. In high vehicle densities the gap become more important ( $\sim 0 \%$ for ViCoV, $18 \%$ for UV-Cast, $31 \%$ for IEEE 1609.4 and $40 \%$ for AID in 400 vehicles $/ \mathrm{Km}^{2}$ ). ViCoV presents largely the lowest frame loss, since it reduces interferences by selecting a sub-set of rebroadcasting vehicles, dynamically selecting the best channel to transmit from the DSRC or CR channels range. The IEEE 1909.4 outperforms AID because it exploits the different service channels of DSRC band to 
transmit video content, while AID transmits using a single channel. However, IEEE 1609.4 does not implement any broadcast suppression mechanism neither a store-carry-forward technic. This is why it presents a higher frame loss than in ViCoV and UV-Cast.

At bitrate of $1 \mathrm{Mbps}$ (Figure 17 (c)), the channel becomes extremely loaded. We note that in this case, the frame loss increases for the protocols UV-CAST, IEEE 1609.4 and AID in high densities scenarios. High frame loss is observed in AID ( 47\%), and IEEE $1609.4(\sim 38 \%)$ at 400 vehicles $/ \mathrm{km}^{2}$, while lightweight frame loss increase is observed in UV-CAST $(\sim 21 \%)$ thanks to broadcast suppression mechanism used in high densities scenarios. On the opposite, $\mathrm{ViCoV}$ performances do not degrade, and we explain this by the interferences avoidance mechanisms implemented by this protocol.

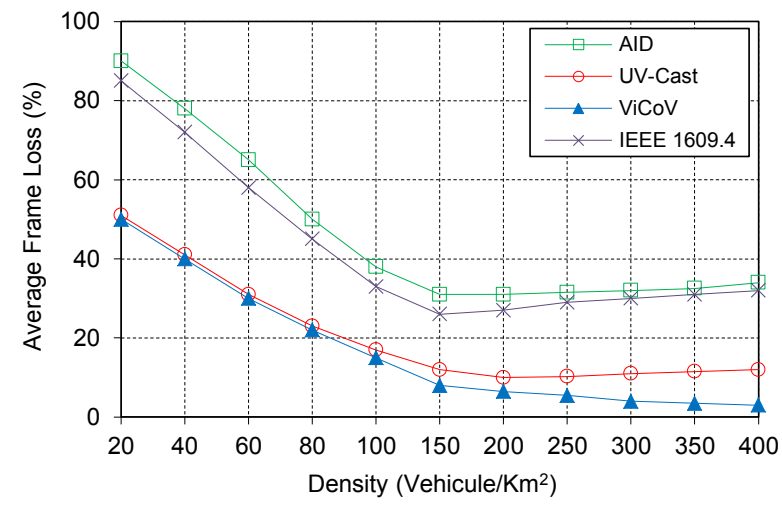

(a) $100 \mathrm{kbps}$

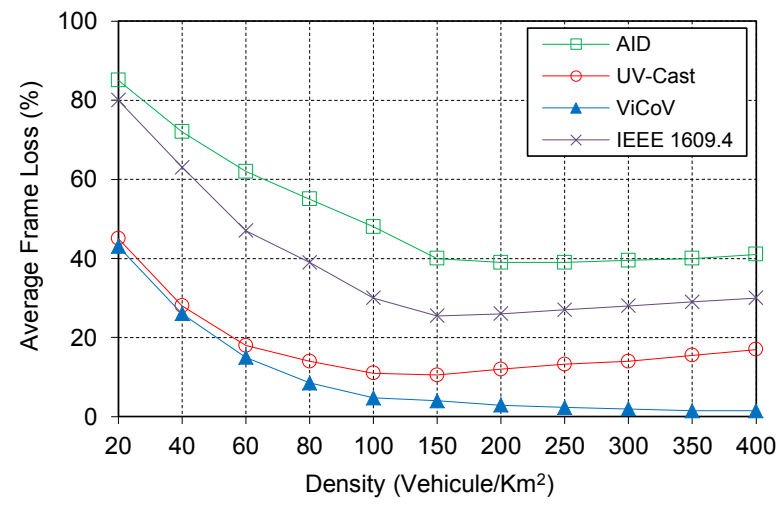

(b) $500 \mathrm{kbps}$

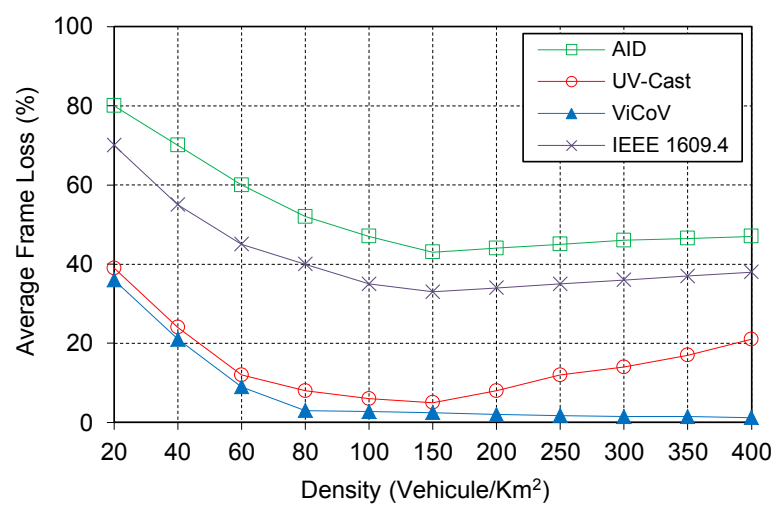

(c) $1 \mathrm{Mbps}$

Figure 17: Average frame loss

\subsection{PSNR}

Figure 18 shows the average Peak Signal-to-Noise Ratio (PSNR) of the reconstructed video at the receivers' vehicles in different vehicle densities scenarios. We remind that a PSNR value higher than or equal to 30 indicates that the video is of a good enough quality.

Figure 18-a shows that at $100 \mathrm{kbps}$, the received video for $\mathrm{ViCoV}$ and UV-cast is of good quality starting from à density of 60 vehicles $/ \mathrm{km}^{2}$. However, this value increases to 100 vehicles $/ \mathrm{km}^{2}$ for AID and IEEE 1609.4. 
At 500 kbps (Figure 18-b), we note a little degradation in PSNR for all the protocols except ViCoV when the vehicle densities become consequent $\left(>150\right.$ vehicles $\left./ \mathrm{Km}^{2}\right)$ due to the high interferences under such conditions.

At $1 \mathrm{Mbps}$, the overall PSNR quality degrades for the four protocols. No one of AID, UV-Cast or IEEE 1609.4 ensures an acceptable reconstructed video quality (PSNR $<30$ ). However, the quality of the received video in $\mathrm{ViCoV}$ is of good quality (PSNR $>30$ ) at density $>=50$ vehicles $/ \mathrm{km}^{2}$, and the gap in PSNR, between ViCoV and the three other protocols, widens in more dense scenarios. For example at 400 vehicles $/ \mathrm{km}^{2}$, the PSNR of the videos delivered by ViCoV is mostly double of the PSNR in the video delivered by UV-Cast. This confirms the effectiveness of the rebroadcaster selection mechanism and the recourse to the $\mathrm{CR}$ channels in dense scenarios proposed in $\mathrm{ViCoV}$.

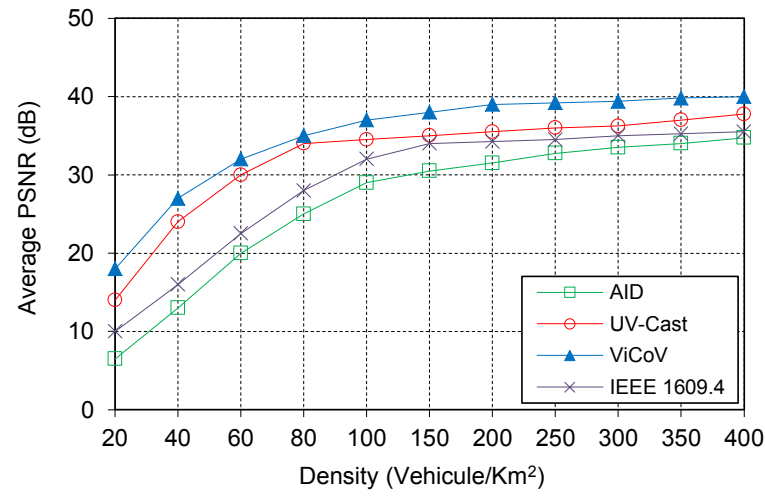

(a) $100 \mathrm{kbps}$

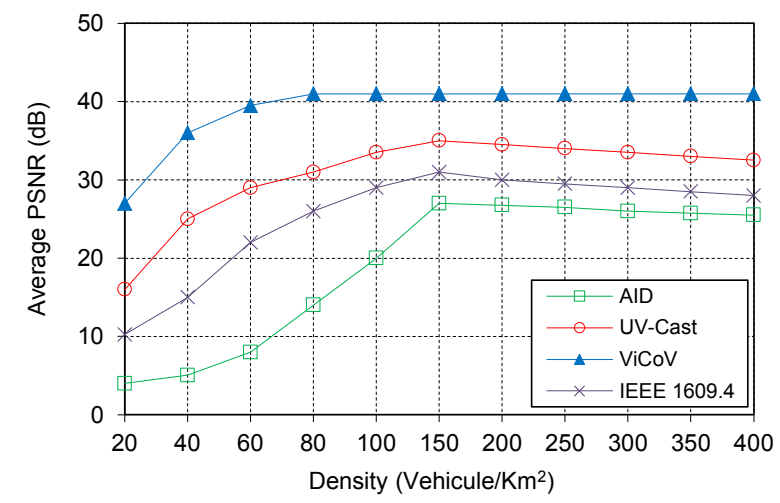

(b) $500 \mathrm{kbps}$

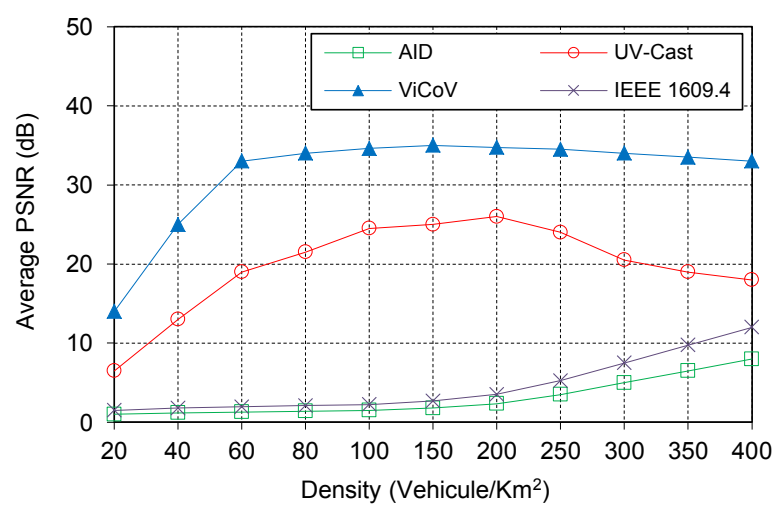

(c) $1 \mathrm{Mbps}$

Figure 18: Peak Signal-to-Noise Ratio

\subsection{Total messages transmitted}

We further studied the total number of data messages sent by vehicles in the four protocols. This metric measures the average of overall messages sent by a vehicle: the initial messages and the retransmitted ones.

The results are represented in Figure 19. We can see that at $100 \mathrm{kbps}$, ViCoV and UV-Cast almost send the same amount of messages. But, when the transmission rate increase to $500 \mathrm{kbps}$ and at high vehicle densities, UV-Cast transmits more messages than $\mathrm{ViCoV}$ although ViCoV performs better frame delivery (see Figure 17). This is can be explained by the fact that more collision are happening in UVCast, and the protocol makes use of store-carry-forward mechanism to recover from collision. We 
remember that, in UV-Cast, all vehicles that are aware of missing messages in the neighborhood retransmit. This increases the number of transmitted messages as well as introduces further collisions. On the other hand, in $\mathrm{ViCoV}$ a minimum set of vehicles (rebroadcaster nodes) retransmit missing messages, and this protocol take advantages of, and select best of, CR channels. The results less messages transmitted in the network and better PSNR and video frames delivery.

Finally, we note that the amount of transmitted messages in AID and IEEE 1609.4 are fewer comparing to UV-Cast and ViCoV. This is due to: a) in low densities, no store-carry-forward mechanism is implemented in AID nor in IEEE 1609.4, consequently less messages circulates in the network; b) in high vehicle densities, UV-Cast and ViCoV retransmit missed messages in the neighbors using the store-carry-forward mechanism which is not implemented in AID and IEEE 1609.4.

We can note that, in low vehicle densities, larger number of total transmitted messages is a good indicator. It shows that the protocol delivers more messages, thanks to the delay tolerant protocol (store-carry-and-forward). This statement can be derived when comparing the performance of ViCoV and UV-Cast against the performances of AID and IEEE1609.4. However, in high vehicle densities, larger number of total transmitted messages can be seen as a bad indicator, since it reflects number of retransmissions performed by a vehicle due to interferences. This statement can be derived from the comparison of the performance of $\mathrm{ViCoV}$ (lower total transmitted messages) and the performance of UV-Cast.

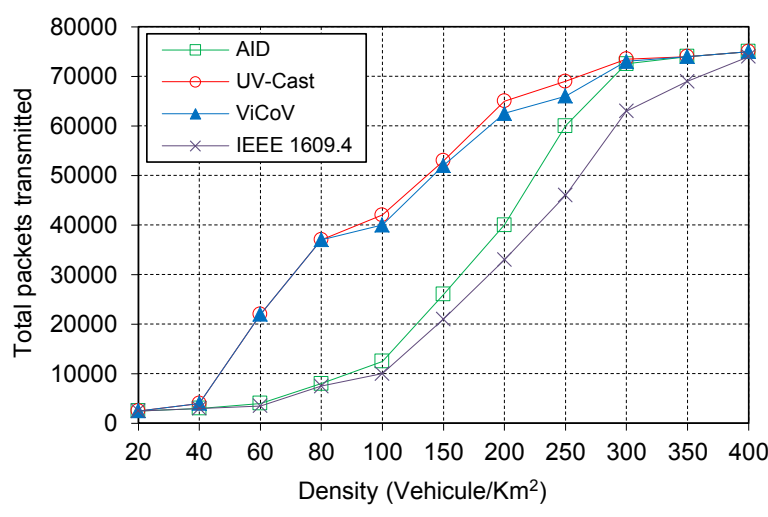

(a) $100 \mathrm{kbps}$

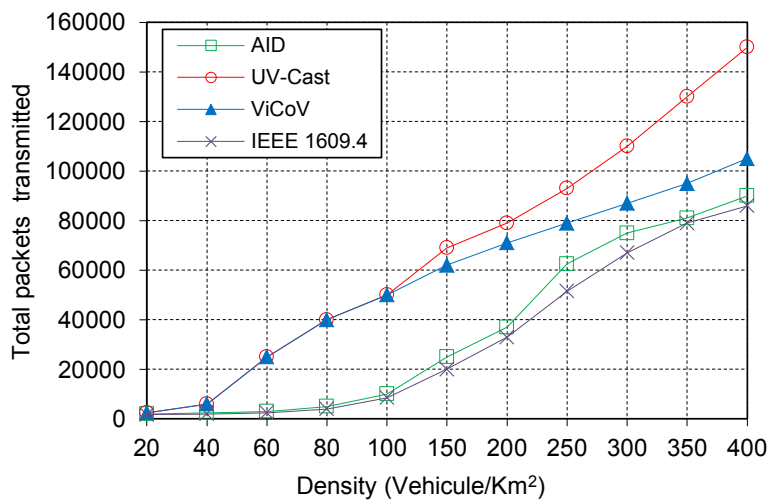

(b) $500 \mathrm{kbps}$

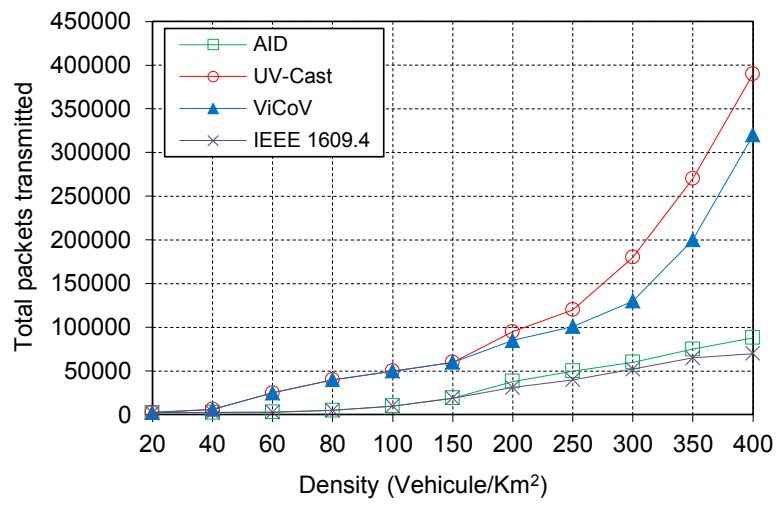

(c) $1 \mathrm{Mbps}$

Figure 19: Total number of content messages transmitted 


\subsection{Frames delay}

In Figure 20, we study the performance of $\mathrm{ViCoV}$ in terms of average delay to deliver frames from the source to receivers.

The main remark is that the frame delay in ViCoV and UV-CAST is more important compared to AID and IEEE 1609.4.

In low densities (20 vehicles $/ \mathrm{km}^{2}$ ), the network is highly intermittent connected. Consequently, only a small proportion of packets are delivered by IEEE 1609.4 and AID. These packets are transmitted directly without any caching mechanism, hence the low delay.

On the opposite, ViCoV and UV-Cast, implement the store-carry-an-forward mechanism and ensure higher delivery ratio, but on the cost of high delay.

We notice also the inversion of the curves shapes for ViCoV and UV-CAST when the density starts to increases (from 20 to 40 vehicles $/ \mathrm{km}^{2}$ ). This behavior is explained by the fact that, when the density starts to increases, the connection time starts to last longer. Thus, more messages are transmitted using the store-carry-forward mechanism, which explains the increase in the average delay. While the density continues to increase ( $>40$ vehicles $\left./ \mathrm{km}^{2}\right)$, the network is more and more connected, and the store-carry-forward mechanism is less used, hence the decrease of the average delay.

We note also that the delay in ViCoV is lower than in UV-CAST, and we explain this by two main factors:

- Less interference in ViCoV implies less retransmission and consequently low frames delay.

- The rebroadcasting selection mechanism in ViCoV (see IV-2 ) selects the vehicle neighbors having high dissemination capacity. This metric is designed in such a way to ensure wide content dissemination in minimum of hops and minimum of retransmissions. Thus, the delay is reduced in ViCoV.

Increasing the bitrate (from 500kbps to $1 \mathrm{Mbps}$ ) leads to reduce the frame loss in ViCoV and UV-Cast as we have seen in Figure 17. This is due in less density scenarios to store-carry-forward mechanism. The consequence is delay decrease with the increase of the bitrates as we can observe in Figure 20-b, c.

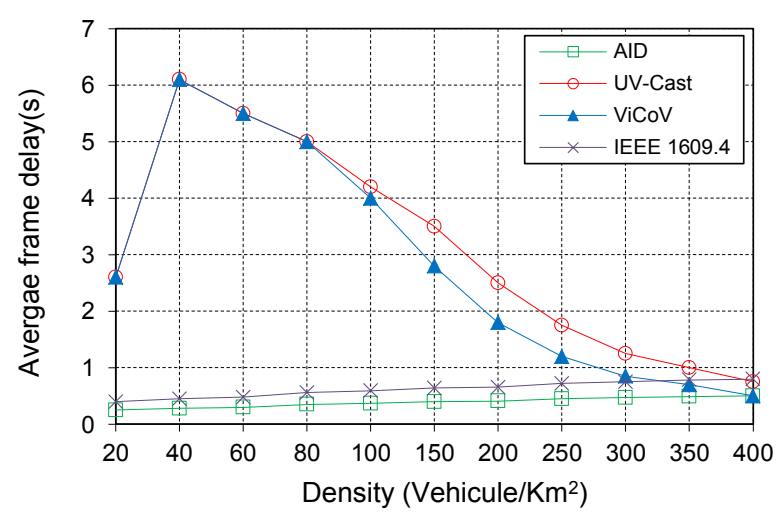

(a) $100 \mathrm{kbps}$

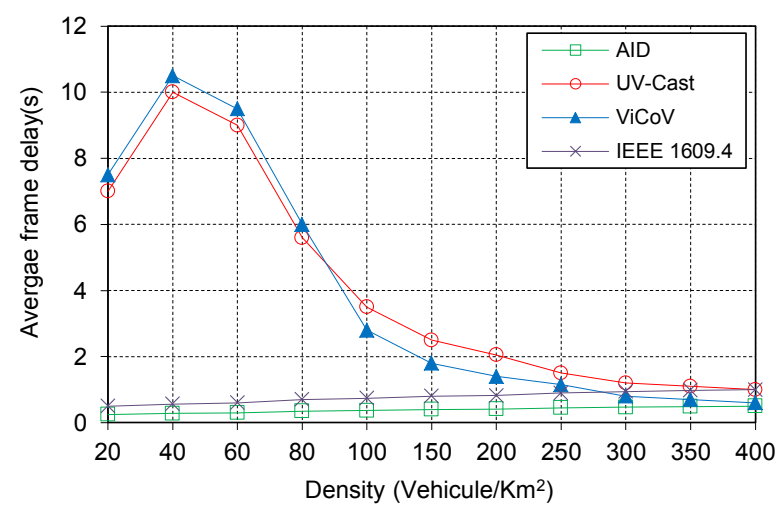

(b) $500 \mathrm{kbps}$ 


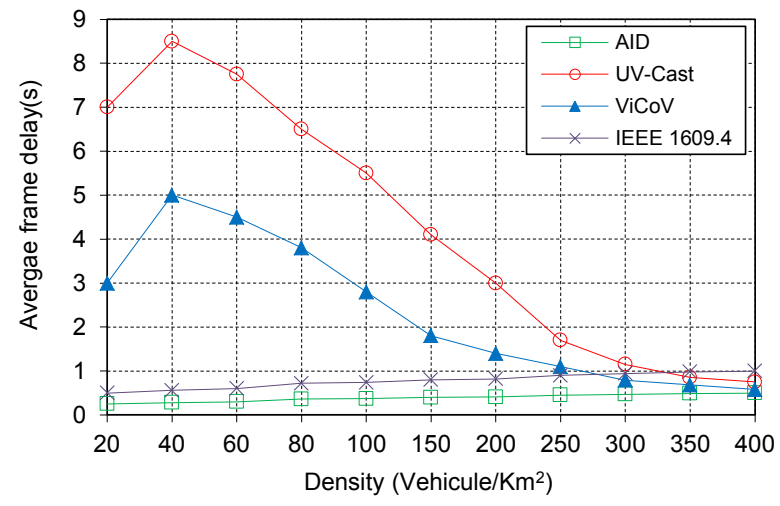

(c) $1 \mathrm{Mbps}$

Figure 20 : Average frame delay

\subsection{Transmission range impact}

We go further in our performance investigation and we study the impact of transmission range on the four protocols (ViCoV, UV-Cast, AID and IEEE 1609.4). For this purpose, we set the transmission range to $500 \mathrm{~m}$ and we measure the metrics: frames loss, PSNR and video frames delay, under a bitrate of $500 \mathrm{kbps}$. The results are compared with the previously obtained ones (transmission range $=300 \mathrm{~m}$ ).

Figure 21 shows the variation of the average frame loss in the four protocols under different vehicle densities for transmission range of $300 \mathrm{~m}$ and $500 \mathrm{~m}$. We note that in low densities scenarios $(<150$ vehicles $/ \mathrm{Km}^{2}$ ), increasing the transmission range reduces the frames loss in the four protocols, which is intuitively explained by the increase in network connectivity. In high densities, increasing the transmission range leads to an increase in the frames loss for UV-Cast, AID and IEEE 1609.4 because of the high interference degree in these conditions. However, we note that ViCoV is not affected. This confirms the effectiveness of the interference avoidance mechanisms proposed in ViCoV.

In Figure 22, we study the average PSNR in the four protocols under transmission range of $300 \mathrm{~m}$ and $500 \mathrm{~m}$. The main remark is that video quality degrades in UV-Cast, AID and IEEE 1609.4 in high densities. For example, at a density of 400 vehicles $/ \mathrm{Km}^{2}$, the PSNR in UV-Cast goes from $33 \mathrm{~dB}$ at transmission range of $300 \mathrm{~m}$ to $25 \mathrm{~dB}$ at a transmission range of $500 \mathrm{~m}$. However $\mathrm{ViCoV}$ is not sensitive to the transmission range increase. The same remark can be derived from Figure 23 regarding the video frames delay which is not affected by the transmission range increase in the case of ViCoV.

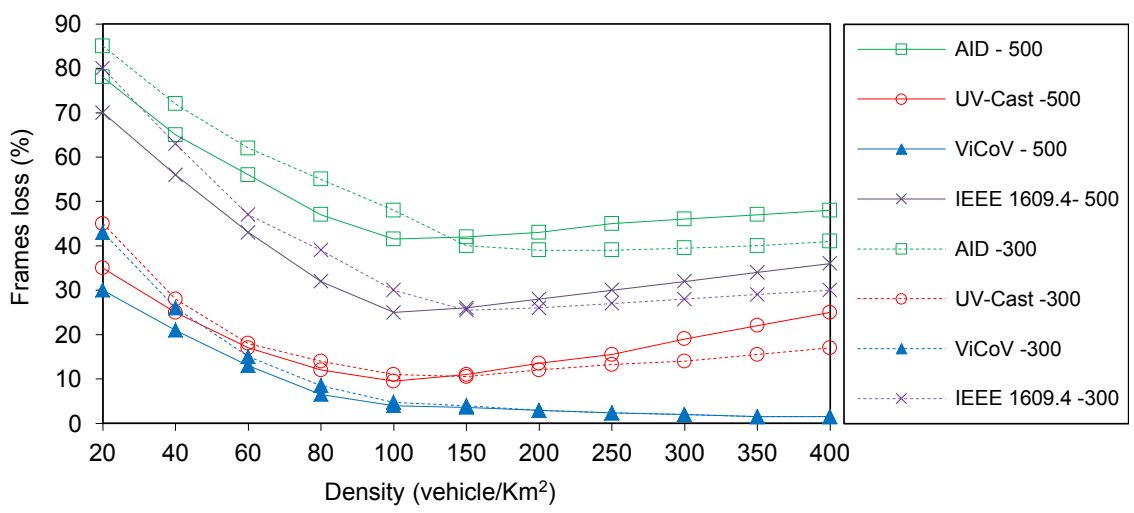

Figure 21: Average frame loss under different transmission ranges 


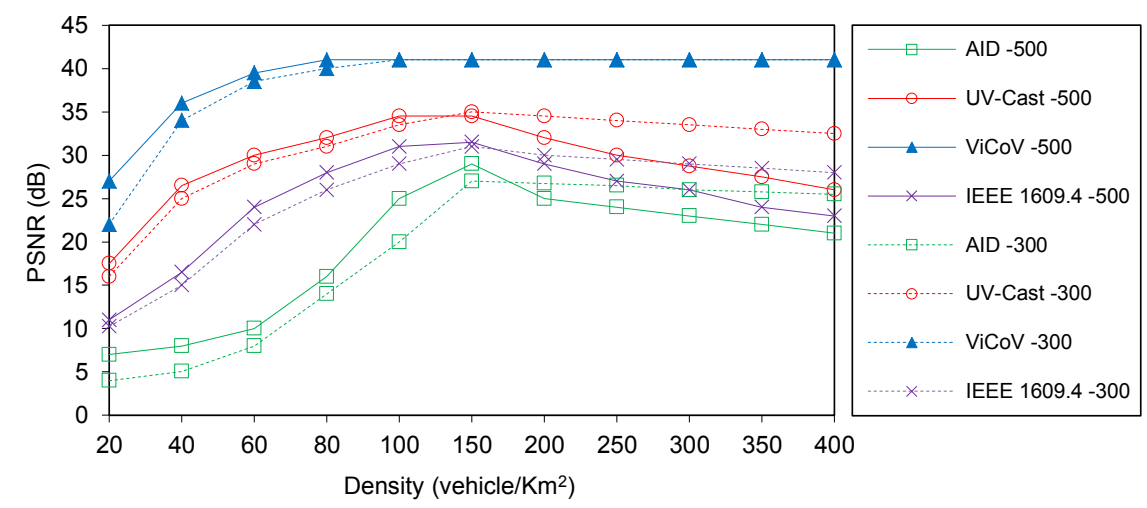

Figure 22: Average PSNR under different transmission ranges

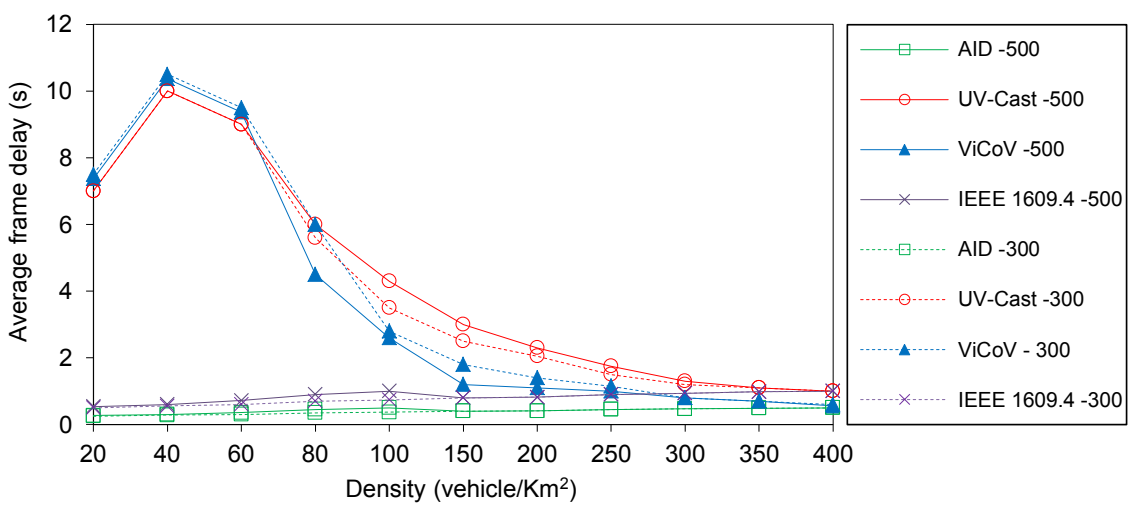

Figure 23: Average frame delay under different transmission ranges

We conclude from this performance study, that $\mathrm{ViCoV}$ is a feasible solution for video streaming over VANETs and its performance outperforms widely those of the pioneering protocols in the state of the art. ViCoV delivered videos are of good quality especially when the vehicle densities is greater than 150 vehicles $/ \mathrm{km}^{2}$. Indeed, in these conditions, the delivered video respect the real time video quality requirement namely a frame loss less than 5\%, frame delay less than 5 seconds and PSNR greater than 30 . We also conclude that a transmission range of $300 \mathrm{~m}$ is sufficient to get these performances.

\section{CONCLUSION}

In this paper, we proposed $\mathrm{ViCoV}$, a video dissemination solution for VANET which operates under different network and traffic conditions. ViCoV selects the best DSRC channel for transmission and exploits the cognitive radio channels to extend the capacity of the network in the dense traffic scenarios. In this case, $\mathrm{ViCoV}$ implements an intelligent mechanism to select the best CR channel using a times series model. In order to avoid the redundant retransmissions, ViCoV selects the most central vehicles in the network to rebroadcast the video stream using a new SNA inspired centrality metric. Furthermore, in the sparse traffic conditions, where the network is usually intermittently connected, vehicles using $\mathrm{ViCoV}$ store the received video content, and deliver it whenever they encounter an uninformed neighbor.

Compared to two related protocols (UV-Cast, AID) and the IEEE 1609.4 standard, ViCoV enhances substantially the quality of the delivered video, in terms of PSNR and frames loss. Indeed, ViCoV reduces the video frame loss to its low values $(<2 \%)$ and increases the stream PSNR by more than $50 \%$ 
compared to the other protocols. The performances of $\mathrm{ViCoV}$ are tangible mostly in dense traffic / high streaming rate scenarios.

In this work we outlined also some scenarios in which $\mathrm{ViCoV}$ is a perfect solution for real time video streaming applications which call for strict quality of service requirements in terms of PSNR, frame loss and frames delay.

\section{REFERENCES}

[1] BENSLIMANE, Abderrahim. Optimized dissemination of alarm messages in vehicular ad-hoc networks (VANET). In : High Speed Networks and Multimedia Communications. Springer Berlin Heidelberg, 2004. p. 655-666.

[2] T. G. P. (2011) Ieee $802.11 \mathrm{p}$ wireless access for vehicular environments. [Online]. Available: http://grouper.ieee.org/groups/802/11/

[3] EICHLER, Stephan. Performance evaluation of the IEEE $802.11 \mathrm{p}$ WAVE communication standard. In : Vehicular Technology Conference, 2007. VTC-2007 Fall. 2007 IEEE 66th. IEEE, 2007. p. 2199-2203.

[4] FCC, Spectrum policy task force report, ET Docket No. 02-135, November 2002.

[5] KATSAROS, Dimitrios, DIMOKAS, Nikos, et TASSIULAS, Leandros. Social network analysis concepts in the design of wireless ad-hoc network protocols. Network, IEEE, 2010, vol. 24, no 6, p. 23-29.

[6] AKYILDIZ, Ian F., LEE, Won-Yeol, et CHOWDHURY, Kaushik R. CRAHNs: Cognitive radio ad hoc networks. Ad Hoc Networks, 2009, vol. 7, no 5, p. 810-836.

[7] UPPOOR, Sandesh et FIORE, Marco. Insights on metropolitan-scale vehicular mobility from a networking perspective. In : Proceedings of the 4th ACM international workshop on Hot topics in planet-scale measurement. ACM, 2012. p. 39-44.

[8] YI, Chih-Wei, CHUANG, Yi-Ta, YEH, Hou-Heng, et al. Streetcast: An urban broadcast protocol for vehicular ad-hoc networks. In : Vehicular Technology Conference (VTC 2010-Spring), 2010 IEEE 71st. IEEE, 2010. p. 1-5.

[9] KORKMAZ, Gökhan, EKICI, Eylem, ÖZGÜNER, Füsun, et al. Urban multi-hop broadcast protocol for inter-vehicle communication systems. In : Proceedings of the 1st ACM international workshop on Vehicular ad hoc networks. ACM, 2004. p. 76-85.

[10] WISITPONGPHAN, Nawaporn, TONGUZ, Ozan K., PARIKH, J. S., et al.Broadcast storm mitigation techniques in vehicular ad hoc networks. Wireless Communications, IEEE, 2007, vol. 14, no 6, p. 84-94.

[11] BAKHOUYA, Mohamed, GABER, Jaafar, et LORENZ, Pascal. An adaptive approach for information dissemination in Vehicular Ad hoc Networks. Journal of Network and Computer Applications, 2011, vol. 34, no 6, p. 1971-1978.

[12] TONGUZ, Ozan K., WISITPONGPHAN, Nawaporn, et BAI, Fan. DV-CAST: A distributed vehicular broadcast protocol for vehicular ad hoc networks. Wireless Communications, IEEE, 2010, vol. 17, no 2, p. 47-57.

[13] UPPOOR, Sandesh et FIORE, Marco. Insights on metropolitan-scale vehicular mobility from a networking perspective. In : Proceedings of the 4th ACM international workshop on Hot topics in planet-scale measurement. ACM, 2012. p. 39-44.

[14] TONGUZ, Ozan K., VIRIYASITAVAT, Wantanee, et BAI, Fan. Modeling urban traffic: a cellular automata approach. Communications Magazine, IEEE, 2009, vol. 47, no 5, p. 142-150.

[15] ZHAO, Jing, ZHANG, Yang, et CAO, Guohong. Data pouring and buffering on the road: A new data dissemination paradigm for vehicular ad hoc networks. Vehicular Technology, IEEE Transactions on, 2007, vol. 56, no 6, p. 3266-3277.

[16] VIRIYASITAVAT, Wantanee, BAI, Fan, et TONGUZ, Ozan K. UV-CAST: an urban vehicular broadcast protocol. In : Vehicular Networking Conference (VNC), 2010 IEEE. IEEE, 2010. p. 25-32. 
[17] DI FELICE, Marco, CHOWDHURY, Kaushik Roy, et BONONI, Luciano. Analyzing the potential of cooperative cognitive radio technology on inter-vehicle communication. In : Wireless Days (WD), 2010 IFIP. IEEE, 2010. p. 1-6.

[18] LI, Husheng, et al. Collaborative spectrum sensing in cognitive radio vehicular ad hoc networks: belief propagation on highway. In : 2010 IEEE 71st Vehicular Technology Conference. 2010. p. 1-5

[19] WANG, Xiao Yu et HO, Pin-Han. A novel sensing coordination framework for CR-VANETs. Vehicular Technology, IEEE Transactions on, 2010, vol. 59, no 4, p. 1936-1948

[20] ZHAO, Jing et CAO, Guohong. Spectrum-Aware Data Replication in Intermittently Connected Cognitive Radio Networks. In : IEEE INFOCOM. 2014

[21] FCC, Notice of proposed rule making and order, ET Docket No. 03-222, December 2003.

[22] HAYKIN, Simon. Cognitive radio: brain-empowered wireless communications.Selected Areas in Communications, IEEE Journal on, 2005, vol. 23, no 2, p. 201-220.

[23] MATINMIKKO, M., MUSTONEN, M., SARVANKO, H., et al. A motivating overview of cognitive radio: Foundations, regulatory issues and key concepts. In : Cognitive Radio and Advanced Spectrum Management, 2008. IEEE CogART 2008.

[24] SENGUPTA, Shamik, HONG, Kai, CHANDRAMOULI, Rajarathnam, et al. Spiderradio: A cognitive radio network with commodity hardware and open source software. Communications Magazine, IEEE, 2011, vol. 49, no 3, p. 101-109.

[25] KHATTAB, Ahmed, PERKINS, Dmitri, et BAYOUMI, M. Design, Implementation and Characterization of Practical Distributed Cognitive Radio Networks. 2013.

[26] PRASANNA, R. et AMRUTUR, Bharadwaj. Cognitive radio implementation for a frequency hopping primary signal. 2013.

[27] LIU, Wei, MEHARI, Michael, BOUCKAERT, Stefan, et al. Demo abstract: a proof of concept implementation for cognitive wireless sensor network on a large-scale wireless testbed. In : 10th European Conference on Wireless Sensor Networks (EWSN-2013). 2013. p. 1-2.

[28] AKYILDIZ, Ian F., LEE, Won-Yeol, VURAN, Mehmet C., et al. NeXt generation/dynamic spectrum access/cognitive radio wireless networks: a survey. Computer Networks, 2006, vol. 50, no 13, p. 2127-2159.

[29] STELlWAGEN, Eric et TASHMAN, Len. ARIMA: The Models of Box and Jenkins. Foresight: The International Journal of Applied Forecasting, 2013, no 30, p. 28-33.

[30] PIÑOL, Pablo, LÓPEZ, Otoniel, MARTÍNEZ, Miguel, et al. Modeling video streaming over VANETs. In : Proceedings of the 7th ACM workshop on Performance monitoring and measurement of heterogeneous wireless and wired networks. ACM, 2012. p. 7-14.

[31] GRAFLING, Sebastian, MAHONEN, Petri, et RIIHIJARVI, Janne. Performance evaluation of IEEE 1609 WAVE and IEEE $802.11 \mathrm{p}$ for vehicular communications. In : Ubiquitous and Future Networks (ICUFN), 2010 Second International Conference on. IEEE, 2010. p. 344-348.

[32] HYNDMAN, Rob J. et KOSTENKO, Andrey V. Minimum sample size requirements for seasonal forecasting models. Foresight, 2007, vol. 6, no Spring, p. 12-15.

[33] CHEN, Qi, JIANG, Daniel, et DELGROSSI, Luca. IEEE 1609.4 DSRC multi-channel operations and its implications on vehicle safety communications. In : Vehicular Networking Conference (VNC), 2009 IEEE. IEEE, 2009. p. 1-8.

[34] Datasheet: AirMagnet Survey - Fluke Networks

[35] AXELL, Erik, LEUS, Geert, LARSSON, Erik G., et al. Spectrum sensing for cognitive radio: State-of-theart and recent advances. Signal Processing Magazine, IEEE, 2012, vol. 29, no 3, p. 101-116.

[36] http://download.mobnets.rwth-aachen.de/

[37] Minitab Inc, 2010. Minitab Statistical Software. Minitab Release 15. http://www.minitab.com.

[38] Priestley, M. B. 1981 Spectral analysis and time series / M.B. Priestley Academic Press, London; New York."OMNeTT++ Discreet Event Simulation System". Internet, 2010 (http://www.omnetpp.org/)

[39] MEDJIAH, Samir et AHMED, Toufik. Orion Routing Protocol for Delay Tolerant Networks. In : Communications (ICC), 2011 IEEE International Conference on. IEEE, 2011. p. 1-6. 
[40] KATSAROS, Dimitrios, DIMOKAS, Nikos, et TASSIULAS, Leandros. Social network analysis concepts in the design of wireless ad hoc network protocols.Network, IEEE, 2010, vol. 24, no 6, p. 23-29.

[41] BAI, Fan et KRISHNAMACHARI, Bhaskar. Exploiting the wisdom of the crowd: localized, distributed information-centric VANETs [Topics in Automotive Networking]. Communications Magazine, IEEE, 2010, vol. 48, no 5, p. 138-146.

[42] UPPOOR, Sandesh et FIORE, Marco. Insights on metropolitan-scale vehicular mobility from a networking perspective. In : Proceedings of the 4th ACM international workshop on Hot topics in planet-scale measurement. ACM, 2012. p. 39-44.

[43] Open Street Map Project: http://www.openstreetmap.org/

[44] The Network Simulator (NS-2), http://www.isi.edu/nsam/ns

[45] SUMO APPLICATION: http://sourceforge.net/apps/mediawiki/sumo/index.php?title=SUMO-User-Documentation

[46] CHAN, An, ZENG, Kai, MOHAPATRA, Prasant, et al. Metrics for evaluating video streaming quality in lossy IEEE 802.11 wireless networks. In : INFOCOM, 2010 Proceedings IEEE. IEEE, 2010. p. 1-9.

[47] http://www2.tkn.tu-berlin.de/research/evalvid/EvalVid/docevalvid.html 